\title{
On the computation of non-perturbative effective potentials in the string theory landscape \\ - IIB/F-theory perspective -
}

\author{
Mirjam Cvetič ${ }^{1,2,3}$, Iñaki García-Etxebarria ${ }^{1,2}$ and James Halverson ${ }^{1,2}$ \\ ${ }^{1}$ Department of Physics and Astronomy, University of Pennsylvania, \\ Philadelphia, PA 19104-6396, USA \\ ${ }^{2}$ Kavli Institute for Theoretical Physics, Kohn Hall, \\ UCSB, Santa Barbara, CA 93106, USA \\ ${ }^{3}$ Center for Applied Mathematics and Theoretical Physics, \\ University of Maribor, Maribor, Slovenia \\ E-mail: cvetic@cvetic.hep.upenn.edu, inaki@sas.upenn.edu, \\ jhal@physics . upenn. edu
}

\begin{abstract}
We discuss a number of issues arising when computing non-perturbative effects systematically across the string theory landscape. In particular, we cast the study of fairly generic physical properties into the language of computability/number theory and show that this amounts to solving systems of diophantine equations. In analogy to the negative solution to Hilbert's 10th problem, we argue that in such systematic studies there may be no algorithm by which one can determine all physical effects. We take large volume type IIB compactifications as an example, with the physical property of interest being the low-energy non-perturbative F-terms of a generic compactification. A similar analysis is expected to hold for other kinds of string vacua, and we discuss in particular the extension of our ideas to F-theory. While these results imply that it may not be possible to systematically answer certain physical questions about generic type IIB compactifications, we identify particular Calabi-Yau manifolds in which the diophantine equations become linear, and thus can be systematically solved.
\end{abstract}

As part of the study of the required systematics of F-terms, we develop technology for computing $\mathbb{Z}_{2}$ equivariant line bundle cohomology on toric varieties, which determines the presence of particular instanton zero modes via the Koszul complex. This is of general interest for realistic IIB model building on complete intersections in toric ambient spaces. 


\section{Contents}

1. Introduction 1

2. Systematics 3

2.1 Neutral zero modes 4

2.2 Charged zero modes 6

2.3 Worldvolume fluxes $\quad 7$

2.4 Generalization to F-theory 8

3. General computability 10

$\begin{array}{lll}3.1 & \text { Formal analysis } & 11\end{array}$

$\begin{array}{ll}3.2 \text { Discussion } & 15\end{array}$

4. Exact superpotentials for special manifolds $\quad 16$

$\begin{array}{lll}4.1 & \text { Factorization exemplified: an elliptic fibration over } \mathbb{P}^{2} & 18\end{array}$

4.2 Further examples: $\mathbb{F}_{n}$ and $d P_{2}$ base, a non-factorizing elliptic threefold, and a non-elliptic factorizing threefold 20

$\begin{array}{ll}\text { 5. Conclusions } & 27\end{array}$

A. $\mathbb{Z}_{2}$ equivariant line bundle cohomology 29

$\begin{array}{ll}\text { A.1 The equivariant holomorphic genus } & 29\end{array}$

A.2 Line bundle cohomology 32

$\begin{array}{llr}\text { A.3 Koszul resolution } & 39\end{array}$

$\begin{array}{lll}\text { A.4 Permutation orientifolds } & 43\end{array}$

B. Factorization: a geometric viewpoint 45

\section{Introduction}

The string theory landscape is vast and unwieldy. In order to understand its dynamics from a top-down perspective, we would like to have a method of constructing the low energy effective action given the compactification data for a string vacuum. In this paper we deal with the question of whether such a method exists using results in computability theory. More precisely, we will describe the computability structure of a well-defined subproblem of the problem of computing low energy effective actions, namely the problem of determining the non-perturbative part of F-terms for all vacua.

There are various reasons to be interested in computing non-perturbative F-terms when building realistic string models. Most prominently, in the context of IIB/F-theory 
compactifications that we study in this paper, D-brane instantons are the only effect that can possibly lift some directions in the classical moduli space that remain flat to all loop orders. The importance of D-brane instantons in the context of Kähler moduli stabilization is well known $[1,2,3]$, and recently it has been realized that the picture is significantly enriched when couplings of instantons to charged zero modes is included in the discussion $[4,5,6,7]$ (for a review see [8]). In particular, couplings in the open string sector that are forbidden in perturbation theory do arise when one takes into account the effect of D-brane instantons. There is another reason why we want to focus on D-brane instantons in this paper. As we will see below, the computability structure of D-brane instantons can be made particularly clear in the context of IIB/F-theory backgrounds, and some interesting links to well known results in number theory arise when looking at things from this perspective.

In particular configurations, exact methods for computing non-perturbative F-terms exist. For example, the low-energy prepotential including all instanton corrections for many $\mathcal{N}=2$ field theories in four dimensions has been obtained exactly using the techniques in $[9,10]$. This can even be reformulated directly in terms of instanton counting [11].

Realistic particle physics theories have at most $\mathcal{N}=1$ supersymmetry in four dimensions, and here the knowledge is more limited. Some $\mathcal{N}=1$ theories admit a matrix model description, and can be solved exactly using the techniques in $[12,13,14]$. This exact solution also sheds light on the problem of classifying instantons contributing to different F-terms [15, 16], a problem that we will discuss extensively below. One can also approach the problem in other ways. For example, one can try to translate known results coming from the topological string to the physical $\mathcal{N}=1$ theory [17, 18], directly orientifold or add fluxes to known $\mathcal{N}=2$ results [19, 20,21, 22, 23, 24], or, in cases where enough of the S-duality group survives, one can use it to constrain the form of the superpotential [25].

The cases where the low energy physics can currently be derived exactly are nevertheless rather special, and one may wonder if anything can be said about the problem in generality, that is, across the whole landscape of string vacua. In this paper we argue that the structure of vacua in the landscape determines whether the problem of determining exact F-terms systematically has a solution or not. The more generic the number theoretical properties of string vacua, ${ }^{1}$ the more likely it is that there is no generic method for computing the low energy effective field theory for an arbitrary compactification.

In order to show this fact, we show that existence of a method for systematically computing the effective field theory for a compactification implies the existence of a method for determining whether a particular class of integer (diophantine) equations derived from the geometric compactification data are solvable. For completely generic diophantine equations, this is known to be impossible to do algorithmically. So we conclude that either:

- The space of possible $\mathcal{N}=1$ vacua of string theory has some hidden algebraic structure, such that the low energy physics can be solved algorithmically, or

- The low energy superpotential for a generic string compactification cannot be computed.

\footnotetext{
${ }^{1}$ We define carefully what we mean by this in section 3 .
} 
To us, the second option seems to be more likely (below we give arguments why), although the first one is also a very interesting possibility, if true, and at the moment we have no way of determining which one is actually realized. In order to give concrete evidence for this result, we will work in a particularly tractable corner of the landscape, namely large volume type IIB compactifications.

Our discussion is organized as follows. In section 2 we will describe the general systematics involved in answering questions about non-perturbative F-terms in large volume type IIB compactifications and their lifts to F-theory. In section 3 we will show a formalization of the approach in section 2 which illuminates the number theoretical structure of the problem (in any approach, not necessarily the one discussed in section 2). In this way we formulate a natural conjecture for the computability structure of the landscape based on known results for very similar problems in number theory. In section 4 we turn the discussion around, showing that a certain class of type IIB Calabi-Yau compactifications admits an algorithm for computing a subset of F-terms systematically. In particular we will see that threefolds with a factorizing intersection form, which include many elliptically fibered Calabi-Yau threefolds, have especially nice properties when it comes to computing superpotentials.

Many of the tools we use in formulating the problem are known (this is the main reason why we chose large volume IIB in the first place), but in order to be completely explicit in our specific computations we needed to develop efficient tools for computing $\mathbb{Z}_{2}$ equivariant line bundle cohomology on toric varieties. We explain how to do this is in appendix A. Finally, appendix B contains a reformulation of part of the discussion in section 4 in terms of lattice data, which may be illuminating to readers familiar with toric geometry.

\section{Systematics}

In this section we will review some basic tools for analyzing D-brane instanton effects in type IIB compactifications, and in particular we present the sheaf cohomology groups and geometric indices relevant to instanton zero modes. The picture seems to be qualitatively similar in F-theory, and we elaborate on this below. Our emphasis will be on showing the existence of explicit computational methods for determining the zero modes on the instanton. As we will see, in the context of IIB/F-theory compactifications coming from a complete intersection in toric varieties there is a systematic method for computing the spectrum of instanton zero modes which can be completed in finite time. The existence of this method will be important in section 3. For an extensive review of D-brane instantons in type II string theory, see [8].

We will be mostly dealing with euclidean D3-branes in type IIB orientifolds. In particular, we will discuss configurations with $\mathrm{O}^{-}$planes, focusing on BPS instantons coming from euclidean D3 branes wrapping holomorphic divisors of the Calabi-Yau. The zero modes of a euclidean D3 brane in such a background naturally split into neutral modes and modes charged under the background space-filling D7 branes. Neutral zero modes are those that do not have charge under the D7 gauge group, while charged modes transform in the (anti)fundamental representation. They arise from open strings going from the in- 
stanton to itself and open strings going from the instanton to the D7, respectively. Let us study each class in turn.

\subsection{Neutral zero modes}

Each BPS D-brane instanton is 1/2 BPS, and thus locally breaks 4 out of the 8 supersymmetries of the background Calabi-Yau geometry. These broken supersymmetries manifest themselves in the volume of the instanton as Goldstinos, that is, as fermionic zero modes. These are conventionally denoted as $\theta^{\alpha}$ and $\bar{\tau}_{\dot{\alpha}}$. Depending on the divisor wrapped by the instanton, some other neutral zero modes, such as deformation modes, may be present.

In a typical $\mathcal{N}=1$ large volume type IIB compactification there are, in addition to the geometric Calabi-Yau background, various other ingredients such as branes, orientifolds and fluxes. Some of these ingredients will change the spectrum of zero modes. For instance, instantons on top of branes have some of their zero modes lifted by ADHM couplings. Or, if an instanton is mapped to itself under the orientifold involution in such a way that only an $O(1)$ gauge group survives, the $\bar{\tau}_{\dot{\alpha}}$ modes are projected out. Similarly, background closed string fluxes can lift some neutral zero modes of the instanton (see $[27,28,29,30]$ for recent works in this direction). In this paper we will ignore this last possibility, and for

\begin{tabular}{c|c} 
Zero modes & Number \\
\hline$\left(X_{\mu}, \theta^{\alpha}\right)$ & $h_{+}^{0}\left(D, \mathcal{O}_{D}\right)=1$ \\
$\bar{\tau}_{\dot{\alpha}}$ & $h_{-}^{0}\left(D, \mathcal{O}_{D}\right)=0$ \\
$\gamma_{\alpha}$ & $h_{+}^{1}\left(D, \mathcal{O}_{D}\right)$ \\
$\left(\omega, \bar{\gamma}_{\dot{\alpha}}\right)$ & $h_{-}^{1}\left(D, \mathcal{O}_{D}\right)$ \\
$\chi_{\alpha}$ & $h_{+}^{2}\left(D, \mathcal{O}_{D}\right)$ \\
$\left(c, \bar{\chi}_{\dot{\alpha}}\right)$ & $h_{-}^{2}\left(D, \mathcal{O}_{D}\right)$
\end{tabular}

Table 1: Zero mode structure for an $O(1)$ instanton wrapping a connected cycle $D$. We follow the conventions in $[8,26]$. Modes with a spinor index are fermionic. The \pm subindex denotes the parity with respect to the orientifold action. our explicit examples we will construct backgrounds without flux. The general structure for the neutral zero modes of an $O(1)$ instanton was discussed in $[31,5,32,33,34,35,36,8,26]$, and we reproduce it in table 1.

If one is interested in contributions to the superpotential of an $\mathcal{N}=1$ compactification, then the instanton will contribute if it has only the two $\theta^{\alpha}$ zero modes. In this paper we will be interested in the more general situation of computing all F-terms, so instantons with extra zero modes are acceptable and interesting, giving rise to F-terms in the $\mathcal{N}=1$ action with a larger number of fermionic operators than two [37, 38]. In some cases these effects can be rather dramatic, for example the quantum deformation of the moduli space of $N_{f}=N_{c}$ SQCD can be attributed to an instanton with four fermionic zero modes [37].

Nevertheless, the superpotential case is still particularly interesting, so let us elaborate on some index techniques which are useful in this case. Looking to table 1 , and recalling that $H^{i}\left(D, \mathcal{O}_{D}\right)=H_{+}^{i}\left(D, \mathcal{O}_{D}\right) \oplus H_{-}^{i}\left(D, \mathcal{O}_{D}\right)$, we have that a necessary condition for a $O(1)$ instanton to contribute to the superpotential is that:

$$
\chi\left(D, \mathcal{O}_{D}\right)=\sum_{i=0}^{2}(-1)^{i}\left(h_{+}^{i}\left(D, \mathcal{O}_{D}\right)+h_{-}^{i}\left(D, \mathcal{O}_{D}\right)\right)=1
$$


This is easy to compute using the Riemann-Roch formula:

$$
\chi\left(D, \mathcal{O}_{D}\right)=\int_{D} \operatorname{ch}\left(\mathcal{O}_{D}\right) \operatorname{Td}(T D)=\int_{D} \operatorname{Td}(T D)
$$

where $T D$ is the tangent bundle to $D$. In section 4 we will make abundant use of this formula.

Similarly, we also need to have that

$$
\chi^{\sigma}\left(D, \mathcal{O}_{D}\right)=\sum_{i=0}^{2}(-1)^{i}\left(h_{+}^{i}\left(D, \mathcal{O}_{D}\right)-h_{-}^{i}\left(D, \mathcal{O}_{D}\right)\right)=1 .
$$

where $\chi^{\sigma}$ denotes Lefschetz's equivariant genus for the orientifold action $\sigma$. We give a brief introduction to the equivariant genus in appendix A.

An important observation, which helps motivate the abstract study of diophantine equations in section 3, is that both (2.2) and (2.3) give rise to equations for integer unknowns with integer coefficients. More precisely, let us take a basis $\left\{D_{i}\right\}$ of divisors of the Calabi-Yau, and write the cycle wrapped by the instanton as $D=\sum d_{i} D_{i}$, with the $d_{i}$ being integers. Then (2.2) gives an inhomogeneous equation of degree 3 on the $d_{i}$ :

$$
\chi\left(D, \mathcal{O}_{D}\right)=a_{i j k} d_{i} d_{j} d_{k}+b_{i j} d_{i} d_{j}+c_{i} d_{i}=1
$$

with the coefficients $a_{i j k}, b_{i j}, c_{i}$ being rational numbers depending on the geometry (we can immediately obtain an equation with integer coefficients by multiplying both sides of the equation by an appropriate integer). Similarly the equivariant index constraint (2.3) gives an inhomogeneous equation of degree 2 on the $d_{i}$ :

$$
\chi^{\sigma}\left(D, \mathcal{O}_{D}\right)=e_{i j} d_{i} d_{j}+f=1
$$

In general, if one is interested in computing all instantons satisfying these conditions one is, in effect, providing a way of solving this system of coupled diophantine equations. We stress that, as we discuss extensively in section 3, the equivalence between questions about non-perturbative dynamics and systems of diophantine equations runs much deeper than what this straightforward discussion would suggest.

We also want to emphasize that, although all line bundle cohomology and index calculations performed in the paper are done in the context of toric geometry, all of the formulas for indices and sheaf cohomology groups given in this section are not in any way specific to Calabi-Yau manifolds that are complete intersections in toric varieties. We choose this type of Calabi-Yau because calculations are particularly tractable in toric geometry, and we can be precise about the structure of the required computation.

\section{Concrete computation of sheaf cohomology}

We see that the problem of computing the spectrum of neutral zero modes on the instanton reduces to computing equivariant line bundle cohomology. Recently work has been done and computer implementations have been provided [39, 40, 41, 42] which allow for the direct calculation of the (non-equivariant) sheaf cohomology groups when the Calabi-Yau 
is a hypersurface or complete intersection in an ambient toric variety. The basic procedure is to first calculate relevant line bundle cohomology on the ambient space which then determines $H^{i}\left(D, \mathcal{O}_{D}\right)$ via the long exact sequences in cohomology associated with Koszul sequences. It is not very hard to extend this procedure to equivariant cohomology, which we show how to do in appendix A.

While it is always possible to algorithmically perform this computation for a given divisor $D$, the problem of systematically determining all instantons which might contribute to the superpotential requires knowledge of $h_{ \pm}^{i}\left(D, \mathcal{O}_{D}\right)$ for a divisor $D=\sum d_{i} D_{i}$ as an explicit function of the $d_{i}$. For simple examples, one can express this function as a simple polynomial in the $d_{i}$, as done in (for example) [26], but for generic backgrounds this will not be possible. This is due to the fact that calculating line bundle cohomology on the ambient space amounts to counting points in polyhedra in the $M$ lattice of the toric variety, which can be solved analytically for simple polyhedra, but not for generic polyhedra that arise in more complicated toric varieties. The only available representation of the result is then in terms of the algorithm that computes line bundle cohomology. This fact is ultimately the reason why, in order to understand the generic structure of non-perturbative F-terms in string theory, we are lead into the mathematical theory of computability.

\subsection{Charged zero modes}

Starting with $[4,5,6]$ it was realized that D-brane instantons provide a way of obtaining couplings in semi-realistic models that are forbidden in perturbation theory. Typical examples are $\mu$ terms [4], neutrino Majorana masses [4, 5, 43, 35, 44], and $10105_{H}$ couplings [45] in $S U(5)$ GUT models. For a recent systematic study of instanton effects on realistic type II MSSM quivers see [46, 47, 48, 49] and references therein.

The important observation is that, in addition to the open strings going from the instanton to itself, there are also open strings going from the instanton to the background D7 branes. These open strings give rise to zero modes of the instanton transforming in the fundamental or antifundamental of the D7 brane gauge group. These zero modes, in turn, can couple to matter fields on the D7 branes, and when integrating over the instanton zero mode measure this process can induce effective operators involving matter fields in the low energy effective action of the compactification.

This implies that in order to understand the physics of charged zero modes we need to understand the structure of Yukawa couplings in our compactification. In this paper we will sidestep this complication by restricting our explicit examples in section 4 to questions that require the absence of charged zero modes on the instanton. In the general discussion of section 3 such a restriction is not made.

In any case, the spectrum of zero modes is obtained easily as follows. Consider a background D7 brane $A$ and an instanton $D$ such that they wrap different divisors. They intersect over the curve $\mathcal{C}=A \cdot D$. The spectrum of zero modes between the instanton and the brane then comes from the cohomology groups [50, 51]:

$$
(\alpha, \bar{\beta}) \in\left(H^{0}\left(\mathcal{C}, K_{\mathcal{C}}^{1 / 2}\right), H^{1}\left(\mathcal{C}, K_{\mathcal{C}}^{1 / 2}\right)\right)
$$


where $\alpha$ and $\bar{\beta}$ stand for the modes in the fundamental and antifundamental respectively of the D7 brane group, and $K_{\mathcal{C}}$ denotes the anticanonical bundle of $\mathcal{C}$. The appearance of the anticanonical class in these formulas comes ultimately from the fact that branes are classified by K-theory, instead of just cohomology [50]. In the examples in section 4 we will ensure that there are no zero modes by ensuring that either

- $\mathcal{C}=\mathbb{P}^{1}$ and $\chi\left(\mathcal{C}, K_{\mathcal{C}}^{1 / 2}\right)=0$, or alternatively,

- $\mathcal{C}=0$, i.e., there is no intersection.

As we will see in examples below, these conditions give respectively quadratic and linear diophantine equations in the integers $d_{i}$ which parameterize the divisor $D$ which the instanton wraps.

Let us mention for completeness that one can also wrap the D-brane instanton on a cycle $S$ also wrapped by a D7 brane. In this case the formulas above need some modification. The relevant formulas for the charged zero modes between the instanton and the brane are $[52,53]$ :

$$
\begin{aligned}
& \alpha \in H^{0}\left(S, K_{S} \otimes \mathcal{L}\right) \oplus H^{1}(S, \mathcal{L}) \oplus H^{2}\left(S, K_{S} \otimes \mathcal{L}\right) \\
& \bar{\beta} \in H^{0}\left(S, K_{S} \otimes \mathcal{L}^{*}\right) \oplus H^{1}\left(S, \mathcal{L}^{*}\right) \oplus H^{2}\left(S, K_{S} \otimes \mathcal{L}^{*}\right)
\end{aligned}
$$

where $\mathcal{L}$ is the line bundle on the $\mathrm{D} 7$ brane. For the applications in the rest of the paper, these modes have little effect: since they come from cohomology classes they are manifestly included in the discussion in section 3 and, since they are a finite number of instantons in this class that can possibly contribute to the superpotential, they do not affect the discussion in section 4 . We will thus ignore this class of instantons in the remainder of the paper.

\subsection{Worldvolume fluxes}

Although we will not consider this in the explicit examples in this paper, let us mention that worldvolume fluxes are also easily included in our discussion. Let us first discuss $O(1)$ instantons. In this case the bundle on the instanton is essentially trivial, and we can assume that it is in fact trivial for our discussion. The $\mathrm{D} 7$ brane can still have a bundle $B$ on its worldvolume, which can give rise to chirality between the brane and the instanton. More precisely, the spectrum of charged zero modes is now given by:

$$
(\alpha, \bar{\beta}) \in\left(H^{0}\left(\mathcal{C}, B \otimes K_{\mathcal{C}}^{1 / 2}\right), H^{1}\left(\mathcal{C}, B \otimes K_{\mathcal{C}}^{1 / 2}\right)\right)
$$

In the case of $U(1)$ instantons we can also consider putting a bundle $\mathcal{L}$ on the instanton. This introduces some new interesting features into the problem. Notice that the problem now involves new integer variables $l_{i}$, which determine the bundle $\mathcal{L}$, in addition to the $d_{i}$. For $\mathcal{L}$ a line bundle we have that $\mathcal{L}=\mathcal{O}\left(\sum l_{i} D_{i}\right)$, with $D_{i}$ some basis of divisors on the instanton worldvolume. In scanning over all possible instantons in the given background we also need to scan over the $l_{i}$. We may also need to make sure that the instanton that we are considering is BPS, otherwise extra Goldstinos will arise in the neutral sector, and 
the resulting coupling is most naturally understood as a D-term. ${ }^{2}$ This will happen when the flux is primitive:

$$
\int_{D} c_{1}(\mathcal{L}) \wedge(J+i B)=0
$$

with $J$ and $B$ the Kähler form and $B$-field respectively. This equation gives a quadratic equation involving $d_{i}$ and $l_{i}$ :

$$
p_{i j} d_{i} l_{j}=0
$$

This case is slightly different from the ones discussed so far, in that even if $d_{i}, l_{i}$ are integer unknowns, the $p_{i j}$ coefficients are general complex numbers, so we cannot talk of diophantine equations. As we will discuss in section 3 , this problem still admits a diophantine representation, as long as $J+i B$ is a computable real number [55] (see [56] for a recent review of the field). Roughly speaking, computable numbers are those numbers that can be computed term by term in a decimal expansion to arbitrary precision. The subset of computable numbers inside the reals is measure zero, but luckily Kähler moduli arising from moduli stabilization are of this kind, since moduli stabilization amounts to solving some systems of integer equations, which can be done numerically to arbitrary precision. Notice that this idea is general: even if in this paper we center on diophantine systems, we are not restricting ourselves to just integer equations, but rather to equations involving computable numbers, which are in any case those that will appear in satisfactory top-down approaches to physics.

In the presence of the bundle $\mathcal{L},(2.8)$ gets extended in the following way:

$$
(\alpha, \bar{\beta}) \in\left(H^{0}\left(\mathcal{C}, \overline{\mathcal{L}} \otimes B \otimes K_{\mathcal{C}}^{1 / 2}\right), H^{1}\left(\mathcal{C}, \overline{\mathcal{L}} \otimes B \otimes K_{\mathcal{C}}^{1 / 2}\right)\right)
$$

\subsection{Generalization to F-theory}

The previous discussion seems to admit a relatively straightforward lift to F-theory, although some of the details are still under active investigation. For completeness, and since F-theory model building is an active area of current research (starting with [57, 58, 59, 60], see [61] for a review of some of the recent developments), let us sketch how the discussion needs to be modified in this case. In the context of this paper, where we mostly discuss complete intersections on toric ambient spaces, there are systematic methods for uplifting the IIB discussion $[62,63,64,65,26,39]$. One can also gain some understanding when a dual heterotic description is available [66]. In the generic case not coming from a weakly coupled IIB compactification or the heterotic string, the counterparts of some important notions in the discussion above are still incompletely understood, so this review is necessarily incomplete.

\footnotetext{
${ }^{2}$ Sometimes one is free to move in moduli space in such a way that the instanton becomes BPS at some point. In the BPS locus the extra Goldstinos turn into extra unsaturated fermionic zero modes, and the D-term is reinterpreted as a local description of a higher F-term [54, 27].
} 


\section{Neutral zero modes}

Euclidean D3-brane instantons lift to euclidean M5 branes in F-theory wrapping a vertical divisor $D$ of a Calabi-Yau 4-fold $X$. Neutral zero modes on the instanton worldvolume $D$ are in one-to-one correspondence with representatives of the cohomology groups $H^{i}\left(D, \mathcal{O}_{D}\right)$ [67]. See $[26,66]$ for the detailed map between the $H^{i}\left(D, \mathcal{O}_{D}\right)$ and the IIB modes in table 1. The $\theta^{\alpha}$ modes we discussed above live in $H^{0}\left(D, \mathcal{O}_{D}\right)$, which must be 1 in order for the $M 5$ instanton to contribute to the superpotential. One can then construct a simple index formula that encodes a necessary condition for an instanton to contribute to the $4 \mathrm{~d}$ superpotential [67]:

$$
\chi\left(D, \mathcal{O}_{D}\right)=\sum_{i=0}^{3}(-1)^{i} h^{i}\left(D, \mathcal{O}_{D}\right)=1
$$

As in section 2.1 this equation admits an expression as a diophantine equation using the Riemann-Roch formula. Write $D=\sum d_{i} D_{i}$, with $D_{i}$ a basis of divisors of $X$. Then (2.12) ends up being a degree four equation on the $d_{i}$ with rational coefficients

$$
\chi\left(D, \mathcal{O}_{D}\right)=m_{i j k l} d_{i} d_{j} d_{k} d_{l}+n_{i j k} d_{i} d_{j} d_{k}+l_{i j} d_{i} d_{j}+p_{i} d_{i}=1 .
$$

which can be made into an equation with integer coefficients by multiplying both sides by an appropriate integer in order to cancel denominators.

Notice that we expect the space of F-theory compactification to be larger than the space of perturbative IIB compactifications, so the precise details of the problem to solve are different: we are trying to construct an algorithm to solve a larger set of degree four equations than in type IIB.

\section{Charged zero modes and worldvolume fluxes}

The discussion here is limited by our understanding of worldvolume dynamics in theories with dyonic vortices. Instantons that lift straightforwardly from IIB have worldvolume dynamics very similar to their perturbative IIB description, and can be analyzed along similar lines to section $2.2[52,53,26,39]{ }^{3} \quad$ Some cases in which the local geometry includes exceptional singularities can also be analyzed [26], although it is not clear how to build a global description in general cases where mutually non-local zero modes are present (see [65] for a discussion of some of the subtleties involved).

Similarly, worldvolume fluxes on the F-theory instanton are only well understood in the case where a IIB limit exists. It is nevertheless important to remark that there have recently been important advances in understanding the lift of worldvolume fluxes on D7branes, see for example $[69,70,71,72,73]$. Some of these developments may also be applicable to D-brane instanton physics.

\footnotetext{
${ }^{3}$ Claims to the contrary have been recently been made in [66]. There are cases where one can compare strong and weakly coupled descriptions of D-brane instanton effects [68, 15, 16, 26, 39], and those cases support the agreement between the IIB and F-theory descriptions (excepting possible lifting of pairs of zero modes not protected by perturbative shift symmetries). F-theory backgrounds with no weakly coupled limit may be qualitatively different, and the discussion may have to be modified for those.
} 
As we have just seen, while the general structure in F-theory seems to be analogous to the type IIB one, many important details are still being elucidated, and thus in order to be as explicit as possible we refrain from discussing F-theory compactifications in what follows. Nevertheless, once the dust settles and the non-perturbative effects in F-theory become well understood, we expect the F-theory discussion to be essentially equivalent in its computability structure to the IIB discussion. It will thus just strengthen the points we make below by providing a larger variety of vacua to consider.

\section{General computability}

The previous discussion shows that the ingredients involved in answering questions about the non-perturbative F-terms are all of a similar type. In particular, there are important necessary conditions for superpotential contributions that can be encoded in index formulas, and we showed above that these give rise to diophantine equations. One may thus hope that the systematic study of diophantine equations (which are well studied objects in number theory) may shed some light on the general problem of computability in the string theory landscape.

Nevertheless, there are various complications with this viewpoint. First, it is evident that not all questions reduce straightforwardly to the study of diophantine equations. One example we have already mentioned in section 2.3 is the computation of the supersymmetry conditions on an instanton with worldvolume flux. More generally, one may ask questions that do not reduce simply to index formulas, but which do nevertheless make perfect sense as questions about non-perturbative F-terms. In these cases, while index conditions may provide very stringent necessary conditions, the ultimate criterion for computing superpotentials requires computing equivariant line bundle cohomology, and no closed formulas exist in general.

It turns out that diophantine equations do indeed provide a unifying framework in which to ask some general questions about computability, but we need to expand our viewpoint and use some very non-trivial results in number theory. We proceed to do so in this section.

Let us start by discussing a seemingly cumbersome, but ultimately very illuminating way of setting up the problem of computing non-perturbative F-terms in string compactifications. Namely: build the non-perturbative superpotential of the chosen Calabi-Yau background by iterating over the set of BPS instantons on that background, and for each instanton decide which coupling it contributes to.

Needless to say, this approach has a number of drawbacks. Most importantly, the procedure takes infinite time, which makes it unsuitable for any study of the dynamics of the landscape. We could make the procedure take a finite time if we impose some cut-off on the wrapping numbers of the instanton in terms of basis divisors. Physically this approach is easily justified by noticing that instantons have a suppression factor exponential in the wrapping number, so beyond some particular wrapping number non-perturbative effects will not be visible in any finite-precision experiment. This approach by truncation is easy to implement on a computer, and often gives most of the information one requires. 
Nevertheless, one may not be satisfied by this partial answer by truncation, and would desire to have a more systematic procedure for computing non-perturbative F-terms exactly. We are particularly interested in whether such a procedure can exist. It is in the context of answering this question that the connection to diophantine equations really shines, since it gives a description of the problem amenable to analysis using powerful results in number theory.

Before going into the somewhat technical analysis below, let us summarize the main findings in plain and somewhat imprecise language. Suppose that, as one would hope, there exists a technique for computing exact non-perturbative F-terms on arbitrary IIB/Ftheory large volume Calabi-Yau compactifications. We do not require that the procedure is in any way based on solving the conditions of section 2 (or more generally any kind of diophantine equations), only that it is able to answer in finite time any decision problem involving the exact F-terms, such as "Does every Kähler modulus appear at least once in the superpotential?", "Does the superpotential include terms involving the Kähler moduli $T_{1}$ and $T_{2}$ ?", or perhaps more exotically "Is there any four fermion term involving the charged field $X_{1}$ ?".

Depending on the exact set of questions one is interested in asking, the final answer for our computability analysis may be different. We will therefore be somewhat unspecific in our approach, leaving the set of questions arbitrary. Given a suggested procedure for computing non-perturbative F-terms, one has the set of questions that the procedure can answer, and thus the analysis below will encode whether the procedure can be extended to the whole set of large volume IIB/F-theory vacua. In order to be specific, though, we will have in mind a procedure for computing non-perturbative F-terms such that it can solve in finite time any decision problem involving non-perturbative F-terms. ${ }^{4}$

We will argue below that a procedure applicable to the entire landscape is unlikely to exist, given some genericity conditions on the space of string compactifications. The argument will be a refinement and formalization of the following. Consider any such procedure. As we saw in section 2, such a procedure furnishes a way of determining whether a certain system of integer equations has a solution. Assume the set of equations is generic enough, such that a procedure for determining whether an element of the set has a solution exists if and only if there is a procedure for determining whether an arbitrary system of integer equations has a solution. It is known, due to the negative solution to Hilbert's 10th problem (which we review below), that no such procedure exists for determining solvability of arbitrary integer equations.

\subsection{Formal analysis}

The previous paragraph gives the gist of the argument, but it is rather imprecise. In this section we will formalize the argument and give a well defined characterization of the problem of computability of F-terms. In order to make the conjecture precise we need to introduce some background material in computability theory and formal logic. A very readable review with further references can be found in [74].

\footnotetext{
${ }^{4}$ Decision problems are questions having a well defined "yes" or "no" answer. The class of physical questions is generically richer than this, but decision problems suffice for our analysis.
} 


\section{Diophantine sets}

Recall that a diophantine equation (over the integers) is a polynomial equation with integer coefficients such that the unknowns are also integer numbers. A famous example is:

$$
x^{n}+y^{n}=z^{n}
$$

with $x, y, z \in \mathbb{Z}$. In general, we will be dealing with systems of equations of different orders. A couple of important and elementary facts about diophantine equations are that every system of high order equations can be made into a system of quadratic equations by introducing enough new variables, and that every system of equations $f_{1}=\ldots=f_{k}=0$ is equivalent to the single equation $\sum f_{i}^{2}=0$. This implies that, without loss of generality, we can discuss the case of a single diophantine equation of degree four at most.

Let us also introduce the related notion of diophantine set, defined as follows: consider a diophantine equation $f\left(a_{1}, \ldots, a_{n} ; x_{1}, \ldots, x_{m}\right)=0$ such that $a_{i}, x_{i} \in \mathbb{Z}$. The diophantine set $\mathcal{F}$ associated to $f$ is the set of $a_{i}$ such that $f=0$ has a solution. More formally:

$$
\mathcal{F}[f]=\left\{\left(a_{1}, \ldots, a_{n}\right): \exists\left(x_{1}, \ldots, x_{m}\right) \mid f\left(a_{1}, \ldots, a_{n} ; x_{1}, \ldots, x_{m}\right)=0\right\}
$$

Instead of thinking about diophantine sets, it will sometimes be useful to think about properties defining a diophantine set, we refer to this as diophantine properties. As an example, the set $2 \mathbb{Z}$ of even numbers, or equivalently the property Even $(x)=x \in 2 \mathbb{Z}$, admits the diophantine representation:

$$
x \in 2 \mathbb{Z} \Longleftrightarrow \exists y \mid 2 y=x
$$

\section{Recursive and recursively enumerable sets}

It is also useful to introduce the notion of a recursively enumerable set. A set $\mathcal{R}_{\infty}$ is recursively enumerable if there is a classification algorithm such that for each input $x$ it halts if and only if $x \in \mathcal{R}_{\infty}$. An equivalent definition is that it is possible to construct an algorithm that, possibly in infinite time, lists all elements of the set. This definition allows for the classification algorithm to run indefinitely, but in practice, we need the algorithm to be able to finish in a finite amount of time. If a classification algorithm finishing in finite time exists, the set is called recursive (or computable).

We have the following result by Matiyasevich (building on earlier work of Davis, Putnam and Robinson), connecting diophantine and recursively enumerable sets:

Theorem (Matiyasevich 1970). A set is recursively enumerable $\Longleftrightarrow$ it is diophantine.

In what follows we will thus freely switch between diophantine and recursively enumerable when talking about sets. As done above, we can also introduce the notion of a recursive enumerable property $\mathcal{P}$ as a property defining a recursively enumerable set. If the property $\mathcal{P}$ holds for an element $x$, we must be able to determine so in finite time.

We are now in a position to introduce the main two objects of interest: the diophantine set $\mathfrak{C}_{\mathcal{P}}$ of Calabi-Yau spaces defined by the diophantine property $\mathcal{P}$, and the oracle $\mathfrak{P}$. Let us start by $\mathfrak{C}_{\mathcal{P}}$. We think of $\mathcal{P}$ as a recursively enumerable property having to do with the 
non-perturbative F-terms on the Calabi-Yau compactification of interest. The questions about Kähler moduli mentioned in the introduction to this section furnish typical examples of diophantine properties $\mathcal{P}$. In more generality, any question boiling down to the existence of a (finite) set of instantons with the right spectrum of zero modes in a given background is recursively enumerable, ${ }^{5}$ simply by going through the whole spectrum of instantons and verifying using the methods reviewed in section 2 whether the condition is satisfied or not. ${ }^{6}$

Since $\mathcal{P}$ is by construction a diophantine property of the set of compactifications, there is an associated diophantine equation that has solution if and only if the compactification $X$ has the property $\mathcal{P}$. That is,

$$
\mathcal{P}(X) \Longleftrightarrow \exists x_{1}, \ldots, x_{n} \mid d_{(\mathcal{P}, X)}\left(x_{1}, \ldots, x_{n}\right)=0,
$$

where $d_{(\mathcal{P}, X)}$ is a diophantine equation depending on $\mathcal{P}$ and $X$. Equivalently, we can think of $d_{(\mathcal{P}, X)}$ as encoding the algorithm that will finish in finite time if and only if $\mathcal{P}(X)$ holds, which must exist since $\mathcal{P}$ is recursively enumerable. The set $\mathfrak{C}_{\mathcal{P}}$ is then defined as the diophantine set of Calabi-Yau spaces where $\mathcal{P}$ holds. This set is diophantine as a subset of the set of string compactifications $\mathfrak{C}$. Notice that $\mathfrak{C}_{\mathcal{P}}$ thus constructed can also be seen as a $\mathcal{P}$-dependent subset of the set of diophantine equations with solution, which will be important below.

The oracle $\mathfrak{P}$ for the property $\mathcal{P}$ is defined as an object that for each Calabi-Yau space $X$ determines whether or not $X \in \mathfrak{C}_{\mathcal{P}}$ in finite time. In this language, the problem of whether an algorithm exists for computing F-terms exactly can be recast as whether $\mathfrak{C}_{\mathcal{P}}$ is recursive for all $\mathcal{P}$ relevant to $\mathrm{F}$-terms, or equivalently whether an algorithmic oracle $\mathfrak{P}$ exists for all $\mathcal{P}$ relevant to $F$-terms. Equivalent questions about the recursivity of $\mathfrak{C}_{\mathcal{P}}$ are interesting for other physical properties $\mathcal{P}$.

The space $\mathfrak{C}$ of compactifications is somewhat abstract, so let us formulate the problem (equivalently) in terms of diophantine equations. Denote by $\mathfrak{S}_{(\mathcal{P}, X)}$ the set of diophantine equations having a solution coming from string theory and the property $\mathcal{P}$. This set is isomorphic to $\mathfrak{C}_{\mathcal{P}}$, but now we are dealing with solvable diophantine equations $d_{(\mathcal{P}, X)}$ instead of string vacua $X$ as elements of the set. This set of solvable $d_{(\mathcal{P}, X)}$ is a subset of all possible $d_{(\mathcal{P}, X)}$, which we denote $D_{(\mathcal{P}, X)}$ and which is isomorphic to $\mathfrak{C}$. The question of interest is whether $\mathfrak{S}_{(\mathcal{P}, X)}$ is recursive as a subset of $D_{(\mathcal{P}, X)}$ for all $\mathcal{P}$.

\section{Relation to the solution of Hilbert's 10th problem}

As we just discussed, for each property pair $(\mathcal{P}, X)$ we have a diophantine equation $d_{(\mathcal{P}, X)}=$ 0 that will have a solution if and only if $\mathcal{P}(X)$ holds. Consider the set $\mathfrak{S}$ of all diophantine equations having a solution. Let us denote in addition $D$ as the set of all diophantine

\footnotetext{
${ }^{5}$ Though whether a particular instanton contributes to the superpotential or not can be determined in finite time, there are an infinite number of possible instantons, so that going through each one (in the absence of some better algorithm) makes the set recursively enumerable, rather than recursive.

${ }^{6}$ In section 2 we discussed indices in generality, but discussed the calculation of line bundle cohomology only in the case where the geometry is constructible as complete intersections in a toric variety. We can generalize the discussion in this section to arbitrary Calabi-Yau spaces if we assume that there is a general procedure for computing the spectrum of zero modes for any divisor that always finishes in finite time.
} 
equations. It is easy to see that $\mathfrak{S}$ is diophantine in $D$ : for an element $d \in D$ try all possible values of the unknowns in some order, if $d \in \mathfrak{S}$ then this process will eventually halt, and it will not halt if $d \notin \mathfrak{S}$. Our set $\mathfrak{S}_{(\mathcal{P}, X)}$ is a subset of $\mathfrak{S}$. Since we have a number of different inclusions of interest, let us briefly review them. First of all, it is clear that

$$
\mathfrak{S}_{(\mathcal{P}, X)} \subseteq D_{(\mathcal{P}, X)}
$$

and that the inclusion is diophantine. Similarly,

$$
\mathfrak{S}_{(\mathcal{P}, X)} \subseteq \mathfrak{S}
$$

but notice that now $\mathfrak{S}_{(\mathcal{P}, X)}$ is not clearly diophantine as a subset of $\mathfrak{S}$, since we have not specified a way of determining whether a given solvable diophantine equation comes from string theory. If the set of Calabi-Yau spaces is recursive, it follows easily that $\mathfrak{S}_{(\mathcal{P}, X)} \subseteq \mathfrak{S}$ is also recursive. Another way of saying this is that we are assuming that there is in principle a process that can determine in finite time whether a given diophantine equation with solution comes from a pair $(\mathcal{P}, X)$ as constructed above, for fixed $\mathcal{P}$. It is often conjectured that there is a finite number of families of Calabi-Yau threefolds [75], which we take as an assumption. In this case $\mathfrak{S}_{(\mathcal{P}, X)}$ would be trivially recursive in $\mathfrak{S}$, simply by enumeration of the set of string vacua. Similarly, even if the space of all possible compactifications $\mathfrak{C}$ turns out to be infinite, $\mathfrak{S}_{(\mathcal{P}, X)} \subseteq \mathfrak{S}$ is still recursive as long as $\mathfrak{C}$ decomposes into a finite sum of parametric families, since the parametric family to which a diophantine equation belongs can be determined in finite time.

Let us thus assume for the sake of the argument that the space of string theory vacua is recursive. Given a diophantine equation $d \in D$ and a condition $\mathcal{P}$, this also allows one to determine in finite time whether or not $d$ comes from string theory, that is, whether it is one of the diophantine equations $d_{(\mathcal{P}, X)}$. It renders the inclusion

$$
D_{(\mathcal{P}, X)} \subseteq D
$$

recursive and

$$
\mathfrak{S}_{(\mathcal{P}, X)} \subset D
$$

recursively enumerable.

It is easy to see that if a recursively enumerable set $\mathcal{S}$ is recursive then its complement $\overline{\mathcal{S}}$ is recursively enumerable. The converse is also true: if the complement $\overline{\mathcal{S}}$ of a diophantine set $\mathcal{S}$ is diophantine, then $\mathcal{S}$ is recursive. To see this, imagine running the algorithms for determining whether an element $x$ is in $\mathcal{S}$ or $\overline{\mathcal{S}}$ in parallel, one instruction at a time. Since both sets are diophantine, the algorithm will eventually stop and give the correct answer for whether $x \in \mathcal{S}$ or $x \notin \mathcal{S}$.

We are now in a position to make our intuitive discussion about the relation with Hilbert's 10th problem precise. A simple argument [74] shows that $\overline{\mathfrak{S}}$ is not diophantine in $D$, and thus $\mathfrak{S}$ is not recursive. This is the (negative) solution to Hilbert's tenth problem, since it is equivalent to the statement that there is no algorithm which can determine in 
finite time whether or not an arbitrary diophantine equation has a solution. In our context, we are dealing with $\mathcal{P}$-dependant subsets of $\mathfrak{S}$, or equivalently

$$
\overline{\mathfrak{S}} \subseteq \overline{\mathfrak{S}_{(\mathcal{P}, X)}}
$$

The intuitive notion of genericity of the space of Calabi-Yau vacua, and the possible properties $\mathcal{P}$ to ask for, then amounts to the statement that $\overline{\mathfrak{S}_{(\mathcal{P}, X)}}$ is non-diophantine for some $\mathcal{P}$ as a subset of $D$.

Notice that from this viewpoint, this genericity condition seems rather natural, as in scanning over the space of properties $\mathcal{P}$ and the landscape we are scanning over large subsets of the space of possible diophantine equations. A recursive procedure for determining whether $\mathcal{P}$ is true for an arbitrary Calabi-Yau is now encoded as a procedure for determining whether some rather generic diophantine equations have a solution. This leads us to conjecture the following:

Conjecture (Non-computability). $\mathfrak{S}_{(\mathcal{P}, X)}$ is non-recursive as a subset of $D$ for some $\mathcal{P}$.

\subsection{Discussion}

An important assumption above was that the set of relevant string compactifications is somehow enumerable. This is often implicitly or explicitly taken to be true, and in fact there are various reasons to think that the relevant space of geometric compactifications is finite [75]. In string theory, in addition to the background geometry, there are also typically other elements involved in defining the vacuum, such as branes, orientifolds or fluxes. It is also believed that there are large but finite bounds on the number of flux vacua of string theory (see [76] for a review).

Let us briefly consider the case that an enumeration procedure for string vacua is not constructible, even in principle. This would make the formalization above not valid since we cannot conclude anymore that $\mathfrak{S}_{(\mathcal{P}, X)} \subset D$ is diophantine. Nevertheless, at least intuitively, it is hard to imagine an algorithmic oracle $\mathfrak{P}$ existing for answering any question about the structure of elements of a non-diophantine set. So if string vacua were not enumerable in principle this would seem to rather strengthen the case for non-computability.

Notice that the non-computability conjecture came most naturally as a statement about $\mathfrak{S}_{(\mathcal{P}, X)}$ as a subset of $D$, but physically we are rather interested on the properties of $\mathfrak{S}_{(\mathcal{P}, X)}$ as a subset of $D_{(\mathcal{P}, X)}$ instead. It turns out that the non-computability conjecture above implies the non-recursiveness of $\mathfrak{S}_{(\mathcal{P}, X)}$ in $D_{(\mathcal{P}, X)}$ : assume that $\mathfrak{S}_{(\mathcal{P}, X)}$ is recursive as a subset of $D_{(\mathcal{P}, X)}$. Then, given a diophantine equation $d \in D$ we can determine (by assumption of recursivity of $D_{(\mathcal{P}, X)} \subseteq D$ ) in finite time whether it is in $D_{(\mathcal{P}, X)}$ or not. If not, it is obviously not in $\mathfrak{S}_{(\mathcal{P}, X)}$ either. If it is in $D_{(\mathcal{P}, X)}$, by assumption of recursivity of $\mathfrak{S}_{(\mathcal{P}, X)}$ in $D_{(\mathcal{P}, X)}$, we can determine in finite time whether it is solvable or not. Thus, recursivity of $\mathfrak{S}_{(\mathcal{P}, X)}$ in $D_{(\mathcal{P}, X)}$ implies recursivity of $\mathfrak{S}_{(\mathcal{P}, X)}$ in $D$, and thus runs contrary to the non-computability conjecture above. Therefore, if the conjecture holds then it is also true that $\mathfrak{S}_{(\mathcal{P}, X)}$ is non-recursive in $D_{(\mathcal{P}, X)}$.

Finally, we would like to emphasize once more that it is the computability structure of the problem that connects the problem of computing F-terms to the abstract discussion of 
diophantine sets, and not just the fact that some simple problems involving index formulas admit a simple diophantine representation, although this was our original motivation. Any question that one can ask, and whose solution can be encoded in terms of a mechanical procedure on the space of instantons, admits a formulation in the framework of this section. In particular, as we have shown explicitly, any question that can be formulated uniquely in terms of the structure of zero modes of various instantons is part of the set $\mathcal{P}$.

\section{Exact superpotentials for special manifolds}

In the previous section we took a look at physical properties $\mathcal{P}$ across the string theory landscape. One interesting class of properties $\mathcal{P}$ involves questions related to the structure of F-terms. We saw that the generic problem admits a neat formulation in terms of computability theory, and this hints to some general properties of the landscape. An important part of the link is the realization that diophantine equations provide a universal description of the computational structure involved.

In this section we want to look to this result from a complementary angle: instead of assuming generic backgrounds and studying the class of equations that arise, we will impose a particular structure for the equations, such that they are solvable, and ask which parts of the landscape give rise to such kind of equations for a particular property $\mathcal{P}$.

Such a structure would determine solvable $\mathcal{N}=1$ backgrounds, in the sense that one can explicitly solve for all instantons satisfying a given condition. Due to its physical interest, the condition that we aim to solve for is that a given instanton contributes to the uncharged superpotential. We do not give the general criterion necessary for solvability, but give instead an interesting family of solvable backgrounds. The basic property common to this class of solvable manifolds turns out to be that their intersection form on the Calabi-Yau factorizes, by which we mean that there is a divisor which participates in every non-zero triple intersection on the Calabi-Yau. Such intersection forms are commonly seen for elliptically fibered threefolds in Weierstrass form, though it also occurs for manifolds which do not admit an elliptic fibration. We give examples of both situations, as well as an example of an elliptic threefold which does not factorize and a factorizing threefold which is not an elliptic fibration.

At this point we wish to explicitly state the conditions which guarantee the solvability of all uncharged instanton corrections to the superpotential:

Solvability Conditions. (Uncharged superpotential corrections)

Given the set $\mathcal{S}$ of smooth Calabi-Yau compactifications with intersection three-form of type $I_{X}=D f_{2}$ for some divisor $D$ and $f_{2}$ a quadratic polynomial of divisors, and $D_{D 7}$ having no component along $D$, then the set $\mathcal{S}_{\mathcal{P}} \subset \mathcal{S}$ for which any given property $\mathcal{P}$ of the uncharged superpotential holds is recursive.

We remind the reader that the properties $\mathcal{P}$ that we are interested in are those that can be formulated in terms of the zero modes of the instantons on each vacuum. The argument for why these conditions allow for a solvability algorithm is roughly as follows: 
an instanton which generates the uncharged superpotential contribution of interest has no charged zero modes, which requires that it not intersect the $D 7$ branes or that it do so at a $\mathbb{P}^{1}{ }^{7}$ If the solvability conditions are satisfied, the corresponding diophantine equations factorize into a product of two linear diophantine equations, rendering it possible to solve for all divisors which do not intersect the $D 7$ branes or do so at a $\mathbb{P}^{1}$. We make this explicit in non-trivial examples, given by Calabi-Yau elliptic fibrations over $\mathbb{P}^{2}, d P_{2}$ and $\mathbb{F}_{n}$.

In fact, for Calabi-Yau elliptic fibrations there exist a set of conditions which ensure that the intersection form factorizes. For generic manifolds, studying these conditions does not necessarily offer any advantage over studying the intersection form directly, but in the case of elliptic fibrations realized as a hypersurface in a toric variety, the conditions make factorization rather transparent, and also suggests a method for constructing an elliptic Calabi-Yau in Weierstrass form over a given base $\mathcal{B} .^{8}$

Consider a Calabi-Yau elliptic fibration (with section) $\pi: X \rightarrow \mathcal{B}$. We call the StanleyReisner ideal of the base and threefold $S R I_{\mathcal{B}}$ and $S R I_{X}$ respectively, and we denote divisors in the base as $C_{i}$, as they are curves in the threefold. Now suppose that the structure of the fibration is such that it satisfies three basic assumptions:

- $X$ satisfies $h^{1,1}(X)=h^{1,1}(\mathcal{B})+1$, which is equivalent to saying that $\operatorname{Div}(X)$ has one more generator than $\operatorname{Div}(\mathcal{B})$.

- Every generator of $S R I_{\mathcal{B}}$ is also a generator of $S R I_{X}$.

- Linear equivalence of base divisors is preserved in $X$. That is, if $C_{i} \sim C_{j}$, then $\pi^{-1}\left(C_{i}\right) \sim \pi^{-1}\left(C_{j}\right)$.

These conditions are satisfied in many concrete examples. For example, the first condition is always satisfied when $X$ is a hypersurface in a toric variety $\mathcal{A}$ with one more $\mathbb{C}^{*}$ action than the toric variety of the base. Notable examples include fibrations where the fiber is a hypersurface in $\mathbb{P}^{2}$ or $\mathbb{P}_{231}$, which include many Weierstrass fibrations. The second condition can be checked explicitly in each topological phase of the threefold, and the third condition is satisfied by many examples of the kind discussed in this work (see tables 2 , 3 and 4) since the homogeneous coordinates corresponding to $\mathcal{B}$ are not charged under the additional $\mathbb{C}^{*}$ action of $\mathcal{A}$. If these conditions are satisfied, then cubic terms in the intersection ring $\mathcal{I}_{X}$ which involve only pullbacks of divisors of the base must vanish. Thus, any non-vanishing triple intersection of divisors necessarily involves the divisor $K$ corresponding to the additional Kähler modulus of $X$, and this divisor therefore factorizes out of the intersection three-form.

\footnotetext{
${ }^{7}$ Recall from section 2.2 that charged zero modes are counted by the cohomology groups $H^{0}(\mathcal{C}, \mathcal{L})$ and $H^{1}(\mathcal{C}, \mathcal{L})$ for some bundle $\mathcal{L}$ that depends on worldvolume fluxes and $K_{\mathcal{C}}^{\frac{1}{2}}$. Generically such zero modes exist, except in the special case where $\mathcal{C}=\mathbb{P}^{1}$ and $\mathcal{L}=\mathcal{O}(-1)$, as in that case both cohomologies vanish. This is not so exotic, as $D 7$ branes often intersect a divisor at a $\mathbb{P}^{1}$, and in the absence of worldvolume flux $\mathcal{L}=K_{\mathcal{C}}^{\frac{1}{2}}=\mathcal{O}(-1)$.

${ }^{8}$ Throughout this paper all bases $\mathcal{B}$ are toric. It is also possible to construct factorizing manifolds for cases where $\mathcal{B}$ is a hypersurface in a toric variety, and thus the elliptic threefold is a complete intersection Calabi-Yau. In such a way one can construct elliptic threefolds with base $d P_{5}, d P_{6}, d P_{7}$, and $d P_{8}$ which exhibit a factorizing intersection form.
} 


\subsection{Factorization exemplified: an elliptic fibration over $\mathbb{P}^{2}$}

We would now like to show the use of factorization in solving for non-perturbative effects in an example which is non-trivial, ${ }^{9}$ yet does not involve tedious calculations which obscure the point. To this end, let us consider an elliptically fibered Calabi-Yau threefold over $\mathbb{P}^{2}$. The GLSM data of the toric ambient space $\mathcal{A}$ in which the threefold is a hypersurface is given in table 2. This data can be thought of as being constructed by taking a $\mathbb{P}^{2}$ with homogeneous coordinates $s, t$, and $u$ augmented by a $\mathbb{P}_{231}$ with coordinates $x, y$, and $z$. The Calabi-Yau curve in $\mathbb{P}_{231}$ gives in this way the elliptic fiber in a Weierstrass fibration over $\mathbb{P}^{2}$. The GLSM charges of $x, y$, and $z$ under $Q_{1}$ are determined by the Calabi-Yau conditions associated with the Weierstrass equation.

There are two triangulations corresponding to these GLSM charges, one of which has a Stanley-Reisner ideal given by $S R I=\langle s t u, x y z\rangle$, which is the one considered here. The generators of the $S R I$ in this topological phase are thus simply the generators of $S R I_{\mathbb{P}^{2}}$ and $S R I_{\mathbb{P}_{231}}$. We define the generators of $\operatorname{Div}(\mathcal{A})$ to be $H \equiv D_{s}$ and $K \equiv D_{z} \cdot{ }^{10}$ In this basis, the Calabi-Yau hypersurface in $\mathcal{A}$ has divisor class $18 H+6 K$ and the intersection form

\begin{tabular}{c|c|c|c|c|c|c} 
& $s$ & $t$ & $u$ & $x$ & $y$ & $z$ \\
\hline$Q_{1}$ & 1 & 1 & 1 & 6 & 9 & 0 \\
$Q_{2}$ & 0 & 0 & 0 & 2 & 3 & 1
\end{tabular}

Table 2: GLSM charges for a toric ambient space $\mathcal{A}$ whose Calabi-Yau hypersurface is an elliptic fibration over $\mathbb{P}^{2}$.

on the Calabi-Yau is

$$
I_{X}=K\left(H^{2}-3 H K+9 K^{2}\right) .
$$

This data is already enough to compute interesting topological indices, such as the holomorphic genus or the Euler character of a divisor or curve. Doing so requires calculating the appropriate Chern classes from the given information. The ones that we will need are calculated by adjunction to be

$$
c\left(T_{X}\right)=1+102 H^{2}+69 H K+11 K^{2}-1628 H^{3}-1629 H^{2} K-543 H K^{2}-60 K^{3}
$$

and

$$
c\left(T_{D}\right)=1-n H-m K+\left(n^{2}+102\right) H^{2}+(2 n m+69) H K+\left(m^{2}+11\right) K^{2},
$$

where $X$ is the Calabi-Yau and $D=n H+m K$ is a divisor in it. The intersection form on $D$ is given by:

$$
I_{D}=m H^{2}+(n-3 m) H K+(9 m-3 n) K^{2} .
$$

With this information the holomorphic genus is then easily calculated to be:

$$
\chi\left(D, \mathcal{O}_{D}\right)=\frac{1}{12} \int_{D} c_{1}^{2}\left(T_{D}\right)+c_{2}\left(T_{D}\right)=\frac{1}{2}\left(3 m^{3}-3 m^{2} n+m n^{2}-m+6 n\right),
$$

yielding a diophantine equation which is difficult to solve explicitly for the necessary constraint $\chi\left(D, \mathcal{O}_{D}\right)=1$. If this was the only constraint which must be satisfied for an instanton to contribute to the superpotential, the task of identifying all such instantons would be difficult indeed. Luckily, in this case some of the other necessary conditions become simple enough to allow us to solve the problem exactly.

\footnotetext{
${ }^{9}$ More trivial examples would include smooth Calabi-Yau threefolds with a single Kähler modulus, in which the holomorphic genus is a cubic equation in a single variable, and thus the finite set of instantons satisfying $\chi\left(D, \mathcal{O}_{D}\right)=1$ can be algorithmically determined. For example, on the quintic threefold $\chi\left(D, \mathcal{O}_{D}\right)=\frac{5}{12} n\left(2 n^{2}+10\right)$, where $D=n H$, and therefore no instanton contributes to the superpotential in the absence of flux.

${ }^{10}$ We denote by $D_{x_{i}}$ the divisor given by the coordinate $x_{i}$ vanishing. For example, $D_{s}$ denotes the locus $\{s=0\}$. 
Introduce a holomorphic orientifold involution $\sigma: s \mapsto-s$. The only divisor in $\mathcal{A}$ which is pointwise fixed under $\sigma$ is $D_{s}$. This determines the location of an $O 7$ plane, so that $\left[D_{O 7}\right]=H$. Such an object is magnetically charged under the Ramond-Ramond zero form, and this charge must be cancelled in the internal space by the introduction of $D 7$ branes. This tadpole cancellation condition can be expressed in homology as

$$
\sum_{a} N_{a}\left(\left[D_{a}\right]+\left[D_{a}^{\prime}\right]\right)=8\left[D_{O 7}\right]
$$

where $\left[D_{a}\right]$ is the homology class of a divisor wrapped by $N_{a} D 7$ branes and $\left[D_{a}^{\prime}\right]$ is its image under $\sigma$. In this example it should be noted that $\sigma$ acts trivially on homology, and therefore $\left[D_{a}\right]=\left[D_{a}^{\prime}\right]$, though not necessarily pointwise. Given that $\left[D_{O 7}\right]=H$ in this example, one solution to the tadpole cancellation conditions is to have three $D 7$ branes wrapping $D_{s}$ and one $D 7$ brane wrapping $D_{t}$. If our concern here was with model-building, this would give rise to an $S O(6) \times S p(2)$ gauge group, which could be broken to $U(3) \times U(1)$ by turning on fluxes. For our purposes, it suffices to know which divisors the $D 7$ branes wrap.

We now would like to classify instantons in this example which do not have charged zero modes. This occurs when the divisor which an instanton wraps does not intersect any $D 7$ brane or does so at a $\mathbb{P}^{1}$. Generically, an instanton wrapped on $D$ does not intersect any gauge $D 7$ brane if $K_{i} \cdot D \cdot D_{D 7}=0$ for all linearly independent generators $K_{i}$ of $\operatorname{Div}(X)$ and all $D 7$ branes. Since the divisors which the $D 7$ branes wrap in this example are both $H$ homologically, the non-intersection conditions are

$$
\begin{aligned}
& H \cdot D \cdot H=n H^{3}+m H^{2} K=m=0 \\
& K \cdot D \cdot H=n H^{2} K+m H K^{2}=n-3 m=0,
\end{aligned}
$$

which show that all non-trivial divisors intersect a $D 7$ brane.

We now investigate which divisors $D$ intersect a $D 7$ brane at a $\mathbb{P}^{1}$. Since $\mathbb{P}^{1}$ is the Riemann surface of genus zero, a simple way to do this is to calculate $\chi(C)=\int_{C} c_{1}\left(T_{C}\right)=$ $2-2 g$ for $C=D \cdot D_{D 7}$. For a curve which is a complete intersection in a Calabi-Yau threefold this simplifies to

$$
\chi(C)=-D \cdot D_{D 7} \cdot\left(D+D_{D 7}\right)
$$

and the constraint for intersecting at $\mathbb{P}^{1}$ in this example is therefore

$$
\chi(D \cdot H)=m(3 m-2 n-1)=2 .
$$

This is a quadratic inhomogeneous equation in two variables. Generic inhomogeneous equations are hard to solve, but luckily the left hand side of (4.9) factorizes. Since we are solving over the integers, this simplifies the problem considerably: 2 is a prime number, and thus either

$$
\begin{aligned}
m & = \pm 1 \\
3 m-2 n-1 & = \pm 2
\end{aligned}
$$


with the signs on both equations agreeing, or

$$
\begin{aligned}
m & = \pm 2 \\
3 m-2 n-1 & = \pm 1 .
\end{aligned}
$$

Solving these linear equations is straightforward, the possible solutions are $(m, n)=(1,0)$, $(-1,-1),(2,2)$, and $(-2,-3)$. As it is easily verified by substitution in (4.5), the only divisor in this set with $\chi\left(D, \mathcal{O}_{D}\right)=1$ is $(m, n)=(1,0)$. Or, in other words, $D=K$. This is the divisor corresponding to the $\mathbb{P}^{2}$ base of the fibration, and thus we also know that $h^{i}\left(K, \mathcal{O}_{K}\right)=0$ for $i>0$. An analysis of equivariant cohomology (see details in later examples) shows that $h_{+}^{0}\left(K, \mathcal{O}_{K}\right)=1$ and $h_{-}^{0}\left(K, \mathcal{O}_{K}\right)=0$, from which we conclude that this divisor does indeed contribute to the uncharged superpotential.

There is a valuable general lesson to be learned from this exercise. Equation (4.9) was solvable precisely because it factored into the product of two linear terms. By considering (4.8), it is straightforward to see that such factorization is guaranteed to occur if some divisor $K$ factorizes out of the intersection three-form and $D_{D 7}$ has no component along $K$. The latter condition is rather common for Calabi-Yau orientifold compactifications in type IIB with an intersection form that factorizes.

For Calabi-Yau manifolds of this type, this suggests a generic prescription for investigating instanton effects systematically. Given the genus $g$ of $C=D \cdot D_{D 7}$, solve the product of linear equations given by $\chi(C)$ for all divisors $D$ which intersect the $D 7$ brane at a Riemann surface of that genus. Then, check to see if those divisors satisfy other necessary constraints for superpotential contribution, such as $\chi\left(D, \mathcal{O}_{D}\right)=1$. One might be concerned that this is just replacing one problem with another, since the genus $g$ of $C$ could be any non-negative integer. However, such higher genus curves are accompanied by large numbers of charged zero modes, which would give superpotential corrections of high mass dimension. Since such corrections are irrelevant for low energy physics, it makes sense to set an upper bound $k$ on the genus of the curve and solve $\chi(C)=2-2 g$ only for $g \leq k$.

\subsection{Further examples: $\mathbb{F}_{n}$ and $d P_{2}$ base, a non-factorizing elliptic threefold, and a non-elliptic factorizing threefold}

In this section we present more examples to further illustrate the utility of factorization in solving for instanton corrections. We consider elliptic threefolds with $\mathbb{F}_{n}$ and $d P_{2}$ base, where the toric data of the base is augmented by a copy of $\mathbb{P}_{231}$, along with the Calabi-Yau conditions coming from the Weierstrass equation. We also give an example of an elliptic threefold which is a hypersurface in $d P_{1} \times \mathbb{F}_{0}$, where the intersection threeform does not factorize, as can be seen intuitively from the toric data. Finally, we briefly present a wellknown example of a factorizing threefold which admits a $K 3$ fibration, but not an elliptic fibration. 


\section{Elliptic fibrations over $\mathbb{F}_{n}$}

Let us start by discussing elliptic fibrations over $\mathbb{F}_{n} .{ }^{11}$ The relevant GLSM data is given in table 3. We take the triangulation corresponding to $S R I=\langle u v, s t, x y z\rangle$. A hypersurface in the toric variety given by this GLSM data satisfies the conditions for factorization, and indeed we easily find a factorizing intersection form in the Calabi-Yau hypersurface:

$$
I_{X}=P\left(M O-2 M P-n O^{2}+(n-2) O P+8 P^{2}\right) .
$$

We take the holomorphic involution to be $\sigma: v \mapsto-v$ which leaves $D_{v}$ and $D_{u}$ pointwise fixed, so that $\left[D_{O 7}\right]=\left[D_{u}\right]+\left[D_{v}\right]=n M+2 O$.

As one would expect, the branes which must be added to cancel tadpoles are dependent on which Hirzebruch surface is the base. If we consider wrapping $n_{s} D 7$ branes on $D_{s}, n_{u}$ $D 7$ branes on $D_{u}$ and $n_{v} D 7$ branes on $D_{v}$, tadpole cancellation and the wrapping of the $D 7$ branes on effective di-

\begin{tabular}{c|cccc|ccc} 
& $s$ & $t$ & $u$ & $v$ & $x$ & $y$ & $z$ \\
\hline$M$ & 1 & 1 & $n$ & 0 & $2(n+2)$ & $3(n+2)$ & 0 \\
$O$ & 0 & 0 & 1 & 1 & 4 & 6 & 0 \\
\hline$P$ & 0 & 0 & 0 & 0 & 2 & 3 & 1
\end{tabular}

Table 3: GLSM charges for a 4D toric ambient space $\mathcal{A}$ whose Calabi-Yau hypersurface is an elliptic fibration over $\mathbb{F}_{n}$. visors requires $n_{u}+n_{v}=8$ and $n_{s}+n n_{u}=4 n$ with $n_{u} \leq 4$. We choose to have $n_{u}=3$ branes wrapping $D_{u}, n_{v}=5$ branes wrapping $D_{v}$, and $n$ branes wrapping $D_{s}$. This gives rise to an $S O(10) \times S O(6) \times S p(2 n)$ gauge symmetry on the D7 branes.

Writing an arbitrary divisor as $D=m M+o O+p P$, the holomorphic genus is given by:

$$
\begin{gathered}
\chi\left(D, \mathcal{O}_{D}\right)=-\frac{1}{6}\left(3 n o^{2} p-3 n o p^{2}-6 m o p+6 m p^{2}+6 o p^{2}\right. \\
\left.-8 p^{3}+6 n o-12 m-12 o+2 p\right) .
\end{gathered}
$$

Since it again seems rather difficult to solve for all solutions to $\chi\left(D, \mathcal{O}_{D}\right)=1$, we impose the additional constraints coming from requiring the absence of charged zero modes. The conditions for a divisor $D$ to not intersect the $D 7$ branes wrapped on $D_{s}$ are given by

$$
\begin{aligned}
M \cdot D_{s} \cdot D & =0 \\
O \cdot D_{s} \cdot D & =p=0 \\
P \cdot D_{s} \cdot D & =o-2 p=0 .
\end{aligned}
$$

Similar calculations for the intersection of $D$ with the $D 7$ branes on $D_{u}$ and $D_{v}$ give the results

$$
\begin{array}{llll}
D_{s} \cdot D=0 & \leftrightarrow & p=0 & o=0 \\
D_{u} \cdot D=0 & \leftrightarrow & p=0 & m=0 \\
D_{v} \cdot D=0 & \leftrightarrow & p=0 & n o=m .
\end{array}
$$

\footnotetext{
${ }^{11}$ We note that this must be done with some care, as the total space of the fibration has singularities for $n>2$, which would be interpreted in F-theory as non-abelian gauge symmetry.
} 
The conditions for $D$ to intersect the $D 7$ branes wrapping $D_{s}, D_{u}$, or $D_{v}$ at a $\mathbb{P}^{1}$ are respectively given by

$$
\begin{aligned}
& \chi\left(D \cdot D_{s}\right)=-2 p(o-p)=2 \\
& \chi\left(D \cdot D_{u}\right)=p(n p-n-2 m+2 p)=2 \\
& \chi\left(D \cdot D_{v}\right)=p(2 n o-n p+n-2 m+2 p)=2 .
\end{aligned}
$$

Looking to these systems of equations, we again see that these conditions show that $D$ must not intersect any $D 7$ branes or must intersect every one at a $\mathbb{P}^{1}$. Divisors satisfying (4.15) are of the form $D=n N$, with $n$ arbitrary. By substitution in (4.13) we easily see that for such divisors $\chi\left(D, \mathcal{O}_{D}\right)=0$, and thus they do not contribute to the superpotential. On the other hand, $D=P$ satisfies 4.16 , as does $D=-P$ for the special case of $n=0$, that is, when the elliptic fibration has base $\mathbb{F}_{0}=\mathbb{P}^{1} \times \mathbb{P}^{1}$. The latter has $\chi\left(D, \mathcal{O}_{D}\right)=-1$, and therefore does not contribute to the superpotential. For $D=P$, though, $\chi\left(D, \mathcal{O}_{D}\right)=1$.

We have determined that the only instanton which might have the correct uncharged zero mode structure to give rise to a neutral superpotential correction is $D=P$. We now apply the techniques of appendix A to directly calculate the equivariant cohomology $h_{ \pm}^{i}\left(D, \mathcal{O}_{D}\right)$. The relevant Koszul sequences are:

$$
\begin{aligned}
0 & \rightarrow \mathcal{O}_{X}(-D) \rightarrow \mathcal{O}_{X} \rightarrow \mathcal{O}_{D} \rightarrow 0 \\
0 & \rightarrow \mathcal{O}_{\mathcal{A}}(-X) \rightarrow \mathcal{O}_{\mathcal{A}} \rightarrow \mathcal{O}_{X} \rightarrow 0 \\
0 \rightarrow \mathcal{O}_{\mathcal{A}}(-X-D) & \rightarrow \mathcal{O}_{\mathcal{A}}(-D) \rightarrow \mathcal{O}_{X}(-D) \rightarrow 0
\end{aligned}
$$

and thus we must calculate the corresponding ambient space cohomologies. The result is that the only non-zero cohomologies are generated by sections of the form

$$
\begin{aligned}
& H^{0}\left(\mathcal{A}, \mathcal{O}_{\mathcal{A}}\right) \sim \text { const } \\
& H^{4}\left(\mathcal{A}, \mathcal{O}_{\mathcal{A}}(-X)\right) \sim \frac{1}{\text { stuvxyz }} \quad H^{4}\left(\mathcal{A}, \mathcal{O}_{\mathcal{A}}(-X-D)\right) \sim \frac{1}{\text { stuvxy } z^{2}} .
\end{aligned}
$$

Each section contributes once to the corresponding cohomology, so the dimension of each cohomology is one. Utilizing the long exact sequences in cohomology corresponding to the Koszul sequences (4.17) gives $h^{i}\left(D, \mathcal{O}_{D}\right)=(1,0,0)$, in agreement with the holomorphic genus calculation.

Finally, we must calculate how the cohomology $h^{0}\left(D, \mathcal{O}_{D}\right)=1$ splits into equivariant cohomology. Again using the techniques discussed in appendix $A$, the $\mathbb{Z}_{2}$ action on the geometry given by $\sigma: v \mapsto-v$ induces an action on the sections, from which is can be seen that the relevant group character is $\chi_{g}\left(H^{0}\left(\mathcal{A}, \mathcal{O}_{\mathcal{A}}\right)\right)=(1,1)$. This gives that

$$
\chi_{g}\left(H^{0}\left(D, \mathcal{O}_{D}\right)\right)=(1,1),
$$

and thus $h_{+}^{0}\left(D, \mathcal{O}_{D}\right)=1$ and $h_{-}^{0}\left(D, \mathcal{O}_{D}\right)=0$. This indicates that the $\bar{\tau}$ mode has been projected out, as expected since $P$ is an orientifold invariant divisor. From these equivariant cohomology calculations, we see that $P$ satisfies the necessary and sufficient conditions on uncharged zero modes for an instanton wrapping it to give an uncharged superpotential contribution. 


\section{Elliptic fibration over $d P_{2}$}

We now turn to another example of a concrete calculation in a non-trivial case. The GLSM data for this elliptic fibration over $d P_{2}$ is given in table 4 . It is straightforward to see the $d P_{2}$ and $\mathbb{P}_{231}$ structure from the GLSM data, which we have partitioned into the relevant quadrants. (This manifold was constructed in [77] as a del Pezzo transition of $\mathbb{P}_{11169}[18]$.) The intersection form on the Calabi-Yau hypersurface $X$ is given by

$$
I_{X}=P\left(M N+M O-M P-N P-O P-M^{2}-N^{2}-O^{2}+7 P^{2}\right)
$$

Finally, the Stanley-Reisner ideal is given by $S R I=\left\langle x_{1} x_{3}, x_{1} x_{4}, x_{2} x_{3}, x_{2} x_{5}, x_{4} x_{5}, x_{6} x_{7} x_{8}\right\rangle$.

Writing an arbitrary divisor as $D=m M+n N+o O+p P$ and calculating $c\left(T_{D}\right)$ by adjunction as in section 4.1, the holomorphic genus is calculated to be

$$
\begin{aligned}
\chi\left(D, \mathcal{O}_{D}\right)=-\frac{1}{6} & \left(3 m^{2} p-6 m n p+3 n^{2} p-6 m o p+3 o^{2} p+3 m p^{2}\right. \\
& \left.+3 n p^{2}+3 o p^{2}-7 p^{3}-6 m-6 n-6 o+p\right) .
\end{aligned}
$$

We take the orientifold involution to be $\sigma: x_{2} \mapsto-x_{2}$. The divisors which are pointwise fixed by this involution are $D_{2}$ and $D_{5}$ and are thus are wrapped by O7 planes, giving $\left[D_{O 7}\right]=\left[D_{2}\right]+\left[D_{5}\right]=$ $M+2 N$. The Ramond-Ramond tadpole can be cancelled by introducing five $D 7$ branes on $D_{5}$, three on $D_{2}$ and one on $D_{3}$, giving rise to gauge group $S O(10) \times$

\begin{tabular}{c|ccccc|ccc} 
& $x_{1}$ & $x_{2}$ & $x_{3}$ & $x_{4}$ & $x_{5}$ & $x_{6}$ & $x_{7}$ & $x_{8}$ \\
\hline$M$ & 1 & 1 & 1 & 0 & 0 & 6 & 9 & 0 \\
$N$ & 0 & 1 & 0 & 0 & 1 & 4 & 6 & 0 \\
$O$ & 1 & 0 & 0 & 1 & 0 & 4 & 6 & 0 \\
\hline$P$ & 0 & 0 & 0 & 0 & 0 & 2 & 3 & 1
\end{tabular}

Table 4: GLSM charges for a 4D toric ambient space $\mathcal{A}$ whose Calabi-Yau hypersurface is an elliptic fibration over $d P_{2}$.

$S O(6) \times S p(2)$ before turning on fluxes, which can break the first factor to obtain a $S U(5)$ GUT. We wish to examine under what conditions $D$ intersects one of the $D 7$ branes. We again use the techniques described in section 4.1, obtaining the conditions for $D$ to intersect the $D 7$ brane stack on $D_{2}$ :

$$
\begin{aligned}
& M \cdot D_{2} \cdot D=0 \\
& N \cdot D_{2} \cdot D=0 \\
& O \cdot D_{2} \cdot D=p=0 \\
& P \cdot D_{2} \cdot D=o-2 p=0 .
\end{aligned}
$$

Similar calculations for the intersection of $D$ with the $D 7$ branes on $D_{3}$ and $D_{5}$ give the results

$$
\begin{array}{llll}
D_{2} \cdot D=0 & \leftrightarrow & p=0 & o=2 p \\
D_{3} \cdot D=0 & \leftrightarrow & p=0 & m=n+o \\
D_{5} \cdot D=0 & \leftrightarrow & p=0 & m=n .
\end{array}
$$


As before, charged modes can also be absent when $D$ intersects a $D 7$ brane at a $\mathbb{P}^{1}$. Calculating the Euler character for the curves where $D$ intersects each of the divisors which the $D 7$ branes wrap gives the conditions

$$
\begin{aligned}
& \chi\left(D \cdot D_{2}\right)=-2 p(o-p)=2 \\
& \chi\left(D \cdot D_{3}\right)=p(2 m-2 n-2 o+p+1)=2 \\
& \chi\left(D \cdot D_{5}\right)=-p(2 m-2 n-p-1)=2
\end{aligned}
$$

for intersection at a $\mathbb{P}^{1}$. The unique solution for intersecting all three at a $\mathbb{P}^{1}$ is $m=n$, $o=0$ and $p=1$.

Now, the condition for the absence of charged modes is that an instanton wrapped on $D$ must either not intersect each $D 7$ brane or must do so at a $\mathbb{P}^{1}$. Satisfying equation (4.24) requires $p \neq 0$, though, which makes it impossible to satisfy equation (4.23). Thus, the divisors $D$ which have no charged modes either intersect none of the $D 7$ branes or intersect every $D 7$ brane at a $\mathbb{P}^{1}$. The absence of charged modes restricts $D$ to be of the form

$$
D=m M+m N+P
$$

or alternatively

$$
D=m M+m N
$$

where divisors of the first form intersect the $D 7$ branes and divisors of the second form do not. The holomorphic genus of these divisors takes the dramatically simplified form

$$
\begin{aligned}
& \chi\left(D, \mathcal{O}_{D}\right)=m+1=1 \\
& \chi\left(D, \mathcal{O}_{D}\right)=2 m=1
\end{aligned}
$$

Thus, the simple conclusion of these results is that there is no instanton which does not intersect the gauge branes that contributes to the superpotential, and that

$$
D=P=D_{8}
$$

intersects all $D 7$ branes at a $\mathbb{P}_{1}$ and could still contribute to the superpotential, provided it satisfy other necessary constraints. In fact, an instanton wrapping this divisor does satisfy the necessary constraints, and is responsible for generating the $10105_{H}$ Yukawa coupling in a GUT model discussed in [77, 39].

We remind that reader that while it is necessary for an instanton to not intersect the D7 branes or to do so at a $\mathbb{P}^{1}$ in order to have an uncharged superpotential contribution, the latter case is not sufficient. Specifically, if it intersects a $D 7$ brane at a $\mathbb{P}^{1}$, one must explicitly verify whether or not it has charged modes. In the case of the GUT model studied on this manifold, D7 worldvolume fluxes were turned on for the sake of chirality, which greatly affects the charged mode calculation. 


\section{Calabi-Yau hypersurface in $d P_{1} \times \mathbb{F}_{0}$}

We now present a geometry which is qualitatively different from the geometries that we have discussed so far. Up to this point, we have discussed weighted projective spaces and elliptic fibrations, where the latter can be seen as the toric data for some base $\mathcal{B}$ augmented by a copy of $\mathbb{P}_{231}$. Though this choice was natural for an elliptic fibration in Weierstrass form, we now present an example of an elliptically fibered Calabi-Yau threefold with a factorizing intersection form where the toric ambient space does not explicitly contain a copy of $\mathbb{P}_{231}$. Instead of $\mathbb{P}_{231}$, one could augment the base $\mathcal{B}$ by any of the two-dimensional toric varieties represented by the sixteen reflexive two-dimensional polyhedra, as each admits a CalabiYau onefold hypersurface, i.e. an elliptic curve.

For the sake of concreteness, we consider the four-dimensional toric variety $\mathcal{A}=d P_{1} \times \mathbb{F}_{0}$ with $S R I=\langle s u, t v, w x, y z\rangle$ and whose GLSM charges are given in table 5 . The intersection three form on the Calabi-Yau hypersurface $X$ is given by

$I_{X}=2 M N(O+P)-2 N^{2}(O+P)+2 M O P+N O P$.

\begin{tabular}{c|cccccccc} 
& $s$ & $t$ & $u$ & $v$ & $w$ & $x$ & $y$ & $z$ \\
\hline$M$ & 1 & 1 & 1 & 0 & 0 & 0 & 0 & 0 \\
$N$ & 0 & 1 & 0 & 1 & 0 & 0 & 0 & 0 \\
$O$ & 0 & 0 & 0 & 0 & 1 & 1 & 0 & 0 \\
$P$ & 0 & 0 & 0 & 0 & 0 & 0 & 1 & 1
\end{tabular}

Table 5: GLSM charges for the $4 \mathrm{D}$ toric ambient space $d P_{1} \times\left(\mathbb{P}_{1} \times \mathbb{P}_{1}\right)$.

The intersection form does not factorize because $h^{1,1}(X)=4 \neq h^{1,1}\left(d P_{1}\right)+1=2+1$. The fact that conditions on linear equivalence and the Stanley-Reisner ideal are satisfied guarantees that there are no non-zero triple intersections of the pullbacks of the $d P_{1}$ divisors to the ambient space, but here either $O$ or $P$ must participate in every non-zero triple intersection. Thus, we explicitly see the effect of violating the conditions on Kähler moduli necessary for factorization.

We wish to check whether or not this Calabi-Yau threefold admits an elliptic fibration. This occurs [78] when there is an effective divisor $D$ such that:

1. $D \cdot \mathcal{C} \geq 0$ for all curves $\mathcal{C} \in X$

2. $D^{3}=0$

3. $D^{2} \cdot D_{i} \neq 0$ for some divisor $D_{i}$ of $X$.

We take $D=\sum_{i \in\{s, t, u, v\}} \pi^{-1}\left(C_{i}\right)=3 M+2 N$. It is clear from the intersection form that $D^{3}=0$, and moreover it is straightforward to calculate $D^{2} P=16 \neq 0$, so that second and third conditions are satisfied. We must also check that $D \cdot C \geq 0$ for all curves $C$ in the Mori cone. Since in our case elements of the Mori cone can be written as a linear combination of $D_{i} \cdot D_{j}$, it is a sufficient (but not necessary) condition to check that $D \cdot D_{i} \cdot D_{j} \geq 0 \forall i, j \in\{s, t, u, v, w, x, y, z\}$. Direct calculation shows that this is satisfied, and thus this Calabi-Yau threefold admits an elliptic fibration. This example shows explicitly that not all elliptic Calabi-Yau threefolds have a factorizing intersection form.

One might also think that we have presented a boring example, where the elliptic fibration is a trivial since the ambient toric variety is a product space. However, it is not necessarily true that the triviality of an ambient space fibration descends to the fibration 
of some hypersurface. As a counterexample, $d P_{1}$ can be embedded in the product space $\mathbb{P}_{2} \times \mathbb{P}_{1}$, as discussed in appendix A. Similarly, $X$ is a non-trivial elliptically fibered threefold, despite the fact that the toric ambient space is a product space. This can be seen from the fact that a trivial elliptic fibration has $\chi\left(\mathcal{B} \times T^{2}\right)=\chi(\mathcal{B}) \chi\left(T^{2}\right)=0$, but $\chi(X)=-128$.

Finally, it is worth noting one more way in which this manifold is qualitatively different from the others we have considered. In the examples where the toric fibration was non-trivial, as in the case of the ambient spaces for Weierstrass fibrations with the fiber a hypersurface in $\mathbb{P}_{231}$, one could verify that $D_{z}$ was the expected base $\left(\mathbb{P}_{2}, d P_{2}\right.$, et cetera) by computing $K_{D_{z}}^{2}=\int_{D_{z}} c_{1}^{2}\left(T_{D_{z}}\right)$ and $\chi\left(D_{z}\right)=\int_{D_{z}} c_{2}\left(T_{D_{z}}\right)$ by the usual techniques. Here, though, the fact that toric fibration is trivial guarantees that every non-zero triple intersection of divisors must involve at least one of $\{M, N\}$ and at least one of $\{O, P\}$, as is seen explicitly in the intersection three-form. For $D=o O+p P$ we have $K_{D}^{2}=0$, and therefore no divisor of this form is a $d P_{1}$, as one might hope. The divisor $D_{z}$ in the previous examples also would not have been as expected had the toric fibration been trivial.

\section{Factorization without admitting an elliptic fibration}

We present one last example for the purpose of making another qualitative point. So far, we have presented many examples of elliptic threefolds with factorizing intersection form, as well an example of an elliptic threefold where the intersection form does not factorize. In this section we present an example from [79, 80, 81] which is a Calabi-Yau threefold that does factorize, but does not admit an elliptic fibration. Our point in presenting this example, as well as the last one, is to show that though elliptic threefolds furnish many examples of manifolds with factorizing intersection form, not all elliptic threefold factorize, and not all manifolds which factorize are elliptic threefolds.

Consider the particular degree twelve Calabi-Yau hypersurface $X$ in $\mathbb{P}_{11226}$ which was studied in [79] in the context of heterotic-type II duality. Blowing up a singular curve, the intersection numbers on the manifold were calculated in [80] to be

$$
\mathcal{I}_{X}=H\left(4 H^{2}-2 H L\right)
$$

There are only two possible classes of divisors which might satisfy the $D^{3}=0$ condition for an elliptic fibration: those that are multiples of $L$ and those that are multiples of $3 H-2 L$. A curve $l$ was shown to have negative intersection with $D=3 H-2 L$ in [81], where this argument was explicitly made, and therefore this particular $D$ does not satisfy the conditions for the threefold to admit an elliptic fibration. One can see also that $L^{2} F=0$ for all divisors $F$, and therefore $D=L$ does not satisfy the conditions for the threefold to admit an elliptic fibration. Instead, $D=L$ satisfies similar conditions for $X$ to admit a $K 3$ fibration. So, $X$ is an example of a Calabi-Yau threefold with factorizing intersection form which does not admit an elliptic fibration but instead admits a $K 3$ fibration. 


\section{Conclusions}

\section{General conclusions}

In this paper we have discussed some computability features of the physical problem of computing effective potentials in the landscape. As we saw in sections 2 and 3 , the translation of the problem into basic number theoretic terms immediately illuminates rather general features of any potential solution, and in fact suggests that the problem may be algorithmically unsolvable in complete generality in the landscape.

If this observation turns out to be true, then it has important consequences for the study of string vacua. First, more pragmatically, this forces upon us a "patchy" description of the landscape: at any point in time, we will have at our disposal a finite set of tools for studying dynamics in the landscape, which apply only to some sets of string vacua. The non-computability results above would then imply that these tools are necessarily limited: either they do not give exact results in finite time for all well-defined questions, or they do not apply to the whole landscape, only to some particular classes of vacua inside it. An example is our discussion in section 4. There our method of attack was solving the index formulas directly. We saw that algorithms exist for doing so in some generality only for particular classes of spaces, in particular threefolds with factorizing intersection form, which include many elliptically fibered threefolds. In any case, no matter how sophisticated our computational toolkit becomes, our computability conjecture implies that there will always be vacua that are inaccessible to the tools developed so far.

This last observation highlights a more conceptual difficulty arising from our results. Optimistically, it may well happen that our vacuum turns out to be one for which computational techniques are known. Or more physically, if our arsenal of computational tools is large enough, it may be always possible to find computable vacua that match the observed phenomenology to the available accuracy. ${ }^{12}$ Nevertheless, even if we can find such phenomenologically acceptable computable vacua, one of the prominent features of string theory is that it can in principle ask - and answer - questions about why we ended up in our particular vacuum. In other words, we are in principle able to formulate dynamical questions about the landscape. If non-computable vacua abound, and are in a sense generic, this would be telling us that the computational model behind the landscape dynamics is necessarily non-classical. This reinforces the lesson in [82], where it was found that an algorithmic approach to the landscape yields NP-complete problems, and it is hard to imagine how to overcome these in a semi-classical framework. ${ }^{13}$ This is necessarily an important and deep lesson about the dynamics on the landscape.

It could be, of course, that the computability conjecture in section 3 is not true, and the effective potential for an arbitrary string compactification is always exactly computable algorithmically. If such an algorithm was found, we could turn the previous discussion on its

\footnotetext{
${ }^{12}$ This is somewhat similar to the distinction between real numbers and computable real numbers that arose in section 2 .

${ }^{13} \mathrm{~A}$ recent paper exploring related ideas is [83]. There it is argued that some simple statements in a toy version of the landscape are ZFC-undecidable.
} 
head, and by formulating the right questions $\mathcal{P}$ we should be able to map out the landscape in great detail. (This is another reason why we believe that such an algorithmic procedure does not exist.) This would also have interesting repercussions in number theory, providing us with a semi-universal method for solving large classes of very complicated diophantine equations.

\section{Extension of the results to other corners of the landscape}

The discussion in this paper is just a first step, and much remains to be done. We tried to be as inclusive as possible, but also precise in our statements. This leaves room for both expanding the discussion of computability to quantities other than non-perturbative F-terms in large volume type IIB, and for figuring out new classes of backgrounds where (subsets of) the F-terms are exactly computable. We briefly commented on the natural extension to F-theory in section 2.4, but many classes of models other than large volume IIB and F-theory are routinely used in string theory, and it would be rather interesting to understand their computability structure. Let us briefly discuss some classes of string vacua for which we expect similar ideas to apply.

We managed to relate the problem of computing non-perturbative effects to number theory because in IIB/F-theory all necessary computations reduce to algebraic geometry, and in the particular case of complete intersections in toric varieties we could show explicitly the computability structure of the resulting problem in algebraic geometry. The tool of choice in heterotic string model building is generically also algebraic geometry, and thus the study of non-perturbative effects on complete intersections in toric varieties should also have a simple algorithmic characterization there.

Another class of problems that we expect admits a clear computational description is branes located at toric singularities. In this case the description is rather combinatorial, using dimer model techniques [84] (see [85, 86] for reviews). ${ }^{14}$ The effects of D-brane instantons on toric singularities can be described systematically in terms of combinatorial quantities [95, 96, 68]. Use of mirror symmetry then allows us to extend the dimer model discussion to the context of intersecting branes in IIA [97]. As a side remark, since we are dealing with holomorphic quantities one can sometimes connect the large volume approach in this paper directly to the dimer model construction simply by moving in Kähler moduli space [98], in these cases the discussion of this paper applies unchanged (although it will require some translation between the large and small volume descriptions).

\section{Further developments from the number-theoretic perspective}

Our discussion of the number theoretical aspects of the problem was somewhat superficial. There are a number of constructions in which non-computability can be proven, and perhaps an embedding of some of these in string theory can be found. This would definitively show that the landscape is not classically computable. Also, one could devise or adapt

\footnotetext{
${ }^{14}$ Abelian orbifolds are particular cases of toric singularities which also admit a CFT treatment. See for example [87, 34, 88, 89, 24, 90, 91, 92, 93, 94] and references therein for some recent papers discussing D-brane instantons from CFT.
} 
methods for solving the diophantine equations arising from index formulas in backgrounds other than those Calabi-Yau threefolds with factorizing intersection form. As an example of a class of diophantine equations that have been well studied in the mathematics community we would like to highlight elliptic curves. That is, imagine that we are interested in studying instantons contributing to the superpotential, and in computing the necessary condition $\chi\left(D, \mathcal{O}_{D}\right)=1$ we obtain an equation of the form:

$$
y^{2}+a_{1} x y+a_{3} y-x^{3}-a_{2} x^{2}-a_{4} x-a_{6}=0
$$

here the coefficients $a_{i}$ are integers which depend on the geometry of the Calabi-Yau, and $x, y$ are also integers parameterizing the divisor wrapped by the instanton. We are taking $D=x D_{x}+y D_{y}$, with $D_{x}, D_{y}$ two basis divisors of the Calabi-Yau. Equation (5.1) is in fact the equation for an elliptic curve over the integers, and it is known that there are generically just a finite number of integer points $(x, y)$ on (5.1) [99]. From the point of view of this paper, if we had a procedure for obtaining this finite set of points we would have solved the problem of computing superpotentials in this background, since checking the spectrum of zero modes over the finite set of solutions of (5.1) will take a finite time. We are not aware of a general procedure for solving elliptic equations over the integers, but there are known algorithms that work for large numbers of elliptic equations. We refer the reader to [99] for a very readable review of the relevant techniques that also discusses some other classes of potentially relevant diophantine equations.

\section{Acknowledgments}

We would like to acknowledge interesting discussions with Lara Anderson, Matthew Ballard, Andres Collinucci, and Denis Klevers. We gratefully acknowledge the hospitality of the KITP during the Strings at the LHC and in the Early Universe program for providing a stimulating environment during the initial stages of this work. I.G.E. thanks N. Hasegawa for kind support and constant encouragement. This research was supported in part by the National Science Foundation under Grant No. NSF PHY05-51164, DOE under grant DE-FG05-95ER40893-A020, NSF RTG grant DMS-0636606, the Fay R. and Eugene L. Langberg Chair, and the Slovenian Research Agency (ARRS).

\section{A. $\mathbb{Z}_{2}$ equivariant line bundle cohomology}

\section{A.1 The equivariant holomorphic genus}

In addition to the arithmetic genus, there is another set of necessary conditions on contributing instantons coming from Lefschetz's equivariant genus. This is a version of the holomorphic genus that takes into account a possible orientifold involution $\sigma$ :

$$
\chi^{\sigma}(\mathcal{M}, E)=\sum_{i}(-1)^{i}\left(H_{+}^{(0, i)}(\mathcal{M}, E)-H_{-}^{(0, i)}(\mathcal{M}, E)\right)
$$

where $E$ is the bundle (or sheaf) defined over the manifold $\mathcal{M}$ whose genus we are interested in (in general $\mathcal{M}$ is the instanton worldvolume, and $E$ is the trivial sheaf $\mathcal{O}_{\mathcal{M}}$ ), and 
$H_{ \pm}^{(0, i)}(\mathcal{M}, E)$ are the even and odd (under $\sigma$ ) cohomologies of $E$. The Lefschetz equivariant genus formula then states that:

$$
\chi^{\sigma}(\mathcal{M}, E)=\int_{M^{\sigma}} \operatorname{ch}_{\sigma}(E) \frac{\operatorname{Td}\left(T M^{\sigma}\right)}{\operatorname{ch}_{\sigma}\left(\wedge_{-1} \overline{N M^{\sigma}}\right)} .
$$

The various elements entering in this formula require some explanation. ${ }^{15}$ We have denoted by $M^{\sigma}$ the fixed locus in $M$ under the involution $\sigma$. Generically, this fixed locus decomposes as a sum of connected components $M^{\sigma}=\cup C^{\sigma}$. The different components can have different dimensionality, as we will see explicitly in examples below.

$T M^{\sigma}$ and $N M^{\sigma}$ denote the tangent and normal bundles to the fixed locus, respectively, and $\operatorname{Td}\left(T M^{\sigma}\right)$ denotes the Todd class of $M^{\sigma}$ :

$$
\operatorname{Td}\left(T M^{\sigma}\right)=1+\frac{1}{2} c_{1}+\frac{1}{12}\left(c_{1}^{2}+c_{2}\right)+\frac{1}{24} c_{1} c_{2}+\ldots
$$

where $c_{1}$ and $c_{2}$ are the first and second Chern classes of $T M^{\sigma}$.

For a vector bundle $F, \wedge_{-1} F$ denotes the (formal) alternating sum of antisymmetric powers of $F$. As an example, taking $F=\mathcal{L}_{1} \oplus \mathcal{L}_{2} \oplus \mathcal{L}_{3}$, with $\mathcal{L}_{i}$ line bundles, we have that:

$$
\wedge_{-1} F=\mathcal{O}-\mathcal{L}_{1} \oplus \mathcal{L}_{2} \oplus \mathcal{L}_{3}+\left(\mathcal{L}_{1} \otimes \mathcal{L}_{2}\right) \oplus\left(\mathcal{L}_{1} \otimes \mathcal{L}_{3}\right) \oplus\left(\mathcal{L}_{2} \otimes \mathcal{L}_{3}\right)-\mathcal{L}_{1} \otimes \mathcal{L}_{2} \otimes \mathcal{L}_{3}
$$

Finally, $\operatorname{ch}_{\sigma}$ denotes an equivariant Chern character, in the following sense. Consider a line bundle $\mathcal{L}$. Generically, different sections of $\mathcal{L}$ transform with different signs under $\mathbb{Z}_{2}$, but, as it turns out, for each component $C^{\sigma}$ of the fixed locus $M^{\sigma}$ the non-vanishing sections transform with a definite $\operatorname{sign} s_{\sigma}\left(C^{\sigma}, \mathcal{L}\right)= \pm 1$. For each fixed component $C^{\sigma}$ we thus define $\operatorname{ch}_{\sigma}(\mathcal{L})=s_{\sigma}\left(C^{\sigma}, \mathcal{L}\right) \cdot \operatorname{ch}(\mathcal{L})$. A simple way to understand this for toric varieties is the following. Generically, $C^{\sigma}$ is only fixed after applying an appropriate $\mathbb{C}^{*}$ (gauge) transformation. This means that, on the fixed locus $C^{\sigma}$, the $\mathbb{Z}_{2}$ equivariant action embeds in the gauge group of the GLSM. By definition, all sections of $\mathcal{L}$ transform with the same sign under gauge transformations. We will use this viewpoint below to our advantage, as it provides an efficient way of computing $s_{\sigma}\left(C^{\sigma}, \mathcal{L}\right)$.

We will provide many examples of the use of this formula in the next section, when we discuss equivariant line bundle cohomology. From the point of view of this paper, the equivariant holomorphic genus gives an additional necessary constraint which is easily checked with the tools at hand. For example, $H_{+}^{(0,0)}\left(D, \mathcal{O}_{D}\right)$ should be the only nonvanishing cohomology in a IIB compactification without flux if an instanton on $D$ is to contribute to the superpotential, and therefore we need to have that

$$
\chi^{\sigma}\left(D, \mathcal{O}_{D}\right)=1
$$

As an illustration of the techniques, we will check the index formulas for the $O(1)$ instanton candidate found in the elliptic fibration over $d P_{2}$ studied in section 4.2 , which wraps $P=$ $D_{8}$.

\footnotetext{
${ }^{15}$ We would like to thank A. Collinucci for illuminating discussions on eq. (A.2).
} 
Below, when writing the expressions, we will assume that the instanton cycle is not pointwise invariant under the orientifold action, but only curves and (possibly) isolated points inside the instanton are invariant. In the case where the whole instanton is invariant, we have an ordinary gauge instanton for a $U S p\left(2 N_{c}\right)$ or $S O\left(N_{c}\right)$ gauge group, which will only contribute to the superpotential if $N_{c} \geq 0$, and $N_{f}=N_{c}$ or $N_{f}=N_{c}-3$, respectively $[100,101]$.

We will also mostly focus on the case of an instanton wrapping a divisor inside a Calabi-Yau threefold. In this case the general formulas simplify, and the equivariant genus receives contributions only from isolated fixed points (denoted as $O 3$ below) and fixed curves (denoted by $M^{\sigma}$ below). Let us analyze these in turn, starting with isolated fixed points.

\section{Isolated fixed points}

In this case, the general Lefschetz formula gives for each fixed point $O 3$ intersecting $M$ :

$$
\begin{aligned}
\chi^{\sigma}(M, \mathcal{L}) & =\int_{O 3} \operatorname{ch}_{\sigma}(\mathcal{L}) \frac{\operatorname{Td}(T O 3)}{\operatorname{ch}_{\sigma}\left(\wedge_{-1} \bar{N}_{O 3}\right)} \\
& =\int_{O 3} \frac{s_{\sigma}(O 3, \mathcal{L})}{\operatorname{ch}_{\sigma}\left(\mathcal{O}-\bar{N}_{O 3}+\wedge^{2} \bar{N}_{O 3}\right)} \\
& =\int_{O 3} \frac{s_{\sigma}(O 3, \mathcal{L})}{\operatorname{ch}\left(\mathcal{O}+\bar{N}_{O 3}+\wedge^{2} \bar{N}_{O 3}\right)} \\
& =\int_{O 3} \frac{s_{\sigma}(O 3, \mathcal{L})}{1+\operatorname{rk}\left(N_{O 3}\right)+\operatorname{rk}\left(\wedge^{2} N_{O 3}\right)} \\
& =\int_{O 3} \frac{s_{\sigma}(O 3, \mathcal{L})}{1+2+1}=\frac{1}{4} s_{\sigma}(O 3, \mathcal{L})
\end{aligned}
$$

where we have used that the normal bundle is antisymmetric under the involution, that the normal bundle to a point inside a surface has rank two, and that its second antisymmetric power has rank one. ${ }^{16}$ The final formula is thus remarkably simple: each point where the divisor under consideration intersects an isolated $\mathrm{O} 3$ contributes $\pm 1 / 4$ to the equivariant genus, depending on the $\mathbb{Z}_{2}$ character of the fixed point.

In the example which is an elliptic fibration over $d P_{2}$, we have O3 planes at $x_{3}=x_{4}=$ $x_{8}=0$ and at $x_{3}=x_{4}=x_{7}=0$. The first point obviously intersects $D_{8}$, and using the intersection form (4.20) on the Calabi-Yau we have $D_{3} \cdot D_{4} \cdot D_{8}=+1$. The second O3 can be seen not to intersect $D_{8}$ : although $D_{3} \cdot D_{4} \cdot D_{7}$ does intersect $D_{8}$ in the ambient space, this intersection point is outside the Calabi-Yau hypersurface. One easy way to see this is noting that the Calabi-Yau hypersurface has class $3 D_{6}$ and $x_{6} x_{7} x_{8}$ is in the Stanley-Reisner ideal. So in our there is one $O 3$ plane which contributes $\frac{1}{4}$ to $\chi^{\sigma}$ (the character is +1 since we are dealing with the trivial bundle $\mathcal{O}$ ).

\section{Fixed curves}

Fixed curves $M^{\sigma}$ in $M$ also contribute in a simple way to the equivariant genus (see the appendix of [26] for a recent similar discussion in the context of F-theory). Specializing to

\footnotetext{
${ }^{16}$ Locally, write the normal bundle as $N_{O 3}=L_{1} \oplus L_{2}$, and thus $\wedge^{2} N=L_{1} \otimes L_{2}$.
} 
the case of $\mathcal{L}$ being a line bundle and expanding (A.2), we get

$$
\begin{aligned}
\chi^{\sigma}(\mathcal{M}, \mathcal{L}) & =\frac{s_{\sigma}\left(M^{\sigma}, \mathcal{L}\right)}{2} \int_{M^{\sigma}}\left(c_{1}(\mathcal{L})+\frac{1}{2} c_{1}(T M)\right) \\
& =\frac{s_{\sigma}\left(M^{\sigma}, \mathcal{L}\right)}{2} \int_{M^{\sigma}}\left(c_{1}(\mathcal{L})-\frac{1}{2}[M]\right) .
\end{aligned}
$$

Here $[M]$ should be understood as the Poincare dual two-form to $M$ in the ambient CalabiYau $X$, and $T M$ is the tangent bundle to $M$. This expression admits a simple expression in terms of intersection numbers:

$$
\chi^{\sigma}(\mathcal{M}, \mathcal{O})=\frac{s_{\sigma}\left(M^{\sigma}, \mathcal{L}\right)}{2}\left(\mathcal{L}-\frac{1}{2} M\right) \cdot M \cdot \Pi_{O 7}
$$

where $\Pi_{O 7}$ denotes the cycle wrapped by the orientifold planes, and we abuse notation by also writing $\mathcal{L}$ for the divisor associated to the line bundle $\mathcal{L}$.

For our particular example, we have

$$
\begin{aligned}
\chi^{\sigma}\left(D_{8}, \mathcal{O}_{D_{8}}\right) & =-\frac{1}{4} D_{8}^{2} \cdot\left(D_{2}+D_{5}\right) \\
& =-\frac{1}{4} D_{8}^{2} \cdot\left(D_{1}+2 D_{5}\right)=-\frac{1}{4}\left(P^{2} \cdot M+2 P^{2} \cdot N\right)=\frac{3}{4}
\end{aligned}
$$

using the intersection form (4.20). Hence, adding all contributions we have that

$$
\chi^{\sigma}\left(D_{8}\right)=1
$$

so that this instanton satisfies the necessary condition for superpotential contribution coming from Lefschetz's equivariant genus.

\section{A.2 Line bundle cohomology}

In the context of Calabi-Yau spaces constructed as hypersurfaces in toric varieties, one can in fact compute explicitly the equivariant structure of sheaf cohomology, and thus, in particular, the exact spectrum of neutral zero modes on any instanton. The basic tool we will need in order to do this is to compute the action of a $\mathbb{Z}_{2}$ involution on the cohomology of line bundles on the ambient toric variety. One can then use a Koszul sequence to project this down to equivariant instanton cohomology, as we discuss in section A.3.

Consider a toric space $\mathcal{A}$. One can compute line bundle cohomology for a line bundle $\mathcal{L}$ by computing the Čech cohomology of a particular complex built from the local sections of $\mathcal{L}$. We will not need to review the details of the construction here, and we refer the reader instead to the appendix of [39] for a concise review, and [102] for a more systematic exposition. The only point we need from that discussion is that, given a monomial $s=$ $x_{1}^{m_{1}} \cdots x_{n}^{m_{n}}$ describing a local section of $\mathcal{L}$, its contribution to the cohomology can be computed from its behavior on the intersections of open sets in $\mathcal{A}$ (in the sense of whether it is a well defined section in a particular patch or not).

Consider now an involution acting on the coordinates of the GLSM for $\mathcal{A}$ as $x_{i} \mapsto$ $(-1)^{s_{i}} x_{i}$, with $s_{i}$ arbitrary integers. This transformation acts on a well defined way on the local sections, namely:

$$
s=x_{1}^{m_{1}} \cdots x_{n}^{m_{n}} \mapsto(-1)^{s_{1} m_{1}+\ldots+s_{n} m_{n}} x_{1}^{m_{1}} \cdots x_{n}^{m_{n}} .
$$


Notice that this transformation (trivially) does not change the behavior of the local sections on the intersections of opens, in the sense that all local sections in non-equivariant cohomology remain local sections in equivariant cohomology, and the differential maps are unaffected. Thus, the Čech complex determining the contribution of this local section to the sheaf cohomology remains unchanged. So we can understand the map (A.11) as giving the behavior of the $\mathbb{Z}_{2}$ involution on the sheaf cohomology of $\mathcal{A}$. It is clear that this recipe gives the right results for global sections, and since the Cech complex is invariant we believe that it also gives the correct result for local sections corresponding to higher sheaf cohomologies. We have checked that this is true in a large number of examples, some of which we present below.

In order to perform the enumeration of contributing local sections efficiently it is very convenient to use the algorithm described in [40], and proven in [41, 42]. In this language the basic idea is that the $\mathbb{Z}_{2}$ action on each element of the line bundle cohomology is given by the action on the representative rationom. Let us illustrate how this works in a number of simple but illuminating examples.

Projective line: $\mathbb{P}^{1}$

The simplest example that we can consider is $\mathbb{P}^{1}$, with coordinates $\left(x_{0}, x_{1}\right)$. There is a single $\mathbb{Z}_{2}$ involution of this space to consider, namely $\left(x_{0}, x_{1}\right) \rightarrow\left(-x_{0}, x_{1}\right)$. Consider a line bundle $\mathcal{O}(k)$, with $k \geq 0$ for simplicity. The cohomology of this line bundle comes only from its global sections: $H^{n}\left(\mathbb{P}^{1}, \mathcal{O}(k)\right)=0$ for $n>0$, while $H^{0}\left(\mathbb{P}^{1}, \mathcal{O}(k)\right)=k+1$. The sections are given by the monomials:

$$
x_{0}^{n} x_{1}^{k-n} \quad \text { with } 0 \leq n \leq k
$$

The inherited $\mathbb{Z}_{2}$ action on these sections is then simply:

$$
x_{0}^{n} x_{1}^{k-n} \rightarrow(-1)^{n} x_{0}^{n} x_{1}^{k-n}
$$

which induces the corresponding $\mathbb{Z}_{2}$ action on $H^{0}\left(\mathbb{P}^{1}, \mathcal{O}(k)\right)$.

In this simple case the result that we obtain is rather trivially true, since the bundle is generated by its sections, but let us double-check it by computing Lefschetz's holomorphic genus for the line bundle. It is given by:

$$
\chi^{\sigma}(M, \mathcal{L})=\int_{\Phi} \frac{\operatorname{ch}_{\sigma}(\mathcal{L}) \operatorname{Td}(T \Phi)}{\operatorname{ch}_{\sigma}\left(\wedge_{-1} \bar{N} \Phi\right)}
$$

where we have denoted by $\Phi$ the fixed locus of the $\mathbb{Z}_{2}$ action $\sigma$, and the irreducible components of $\Phi$ by $\Phi_{i}$. In the case under study $\sigma$ has two fixed points, $\Phi_{1}=(0,1)$ and $\Phi_{2}=(0,1)$, and the integral splits into two components:

$$
\chi^{\sigma}(M, \mathcal{L})=\int_{(0,1)} \frac{1}{2}+\int_{(1,0)} \frac{(-1)^{k}}{2}=\sum_{i=0}^{k}(-1)^{i} .
$$

The relative factor of $(-1)^{k}$ appears since the character of the line bundle depends on the fixed component under consideration, as explained above. It can be efficiently computed 
as follows: $\Phi_{i}$ may not actually be fixed under the $\mathbb{Z}_{2}$ action $\sigma$ as a subspace of the ambient $\mathbb{C}^{2}=\left\{x_{0}, x_{1}\right\}$ (i.e., before imposing the $\mathbb{C}^{*}$ gauge invariance), and will only become a fixed locus once we quotient $\mathbb{C}^{2}$ by $\mathbb{C}^{*}$. In other words, one may need to combine $\sigma$ with some $\mathbb{C}^{*}$ action $g_{\Phi_{i}}= \pm 1$ in order to leave $\Phi_{i}$ fixed in the ambient space. A bundle with charge $k$ under $\mathbb{C}^{*}$ will then have the character $g_{\Phi_{i}}^{k}$. In our case, we have that $(0,1)$ is fixed by itself under $\sigma$, so its associated character is 1 , while $(1,0)$ requires a -1 action on the covering space, so its character is $(-1)^{k}$. This discussion generalizes straightforwardly to higher dimensions and multiple $\mathbb{C}^{*}$ symmetries, and we just quote the relevant results below.

\section{Projective plane: $\mathbb{P}^{2}$}

For our next example we move one complex dimension higher, and consider $\mathbb{P}^{2}$, parameterized by $\left(x_{0}, x_{1}, x_{2}\right)$. The $\mathbb{Z}_{2}$ orientifold action $\sigma$ to consider in this case is given by:

$$
\left(x_{0}, x_{1}, x_{2}\right) \rightarrow\left(-x_{0}, x_{1}, x_{2}\right)
$$

Let us again consider for simplicity a bundle of the form $\mathcal{O}(k)$, with $k>0$. This bundle is ample, i.e. generated by its sections, and the sections can be described as monomials of the form:

$$
x_{0}^{a} x_{1}^{b} x_{2}^{k-a-b} \quad \text { with } a \geq 0, b \geq 0, k \geq a+b
$$

The action of $\sigma$ is thus given by:

$$
x_{0}^{a} x_{1}^{b} x_{2}^{k-a-b} \rightarrow(-1)^{a} x_{0}^{a} x_{1}^{b} x_{2}^{k-a-b}
$$

inducing the corresponding action on the cohomology.

Let us check this against the result from Lefschetz's theorem. In this case the fixed point set consists of the curve $x_{0}=0$, and the point $(1,0,0)$. By a simple application of (A.14) we obtain:

$$
\chi^{\sigma}\left(\mathbb{P}^{2}, \mathcal{O}(k)\right)=\left(\frac{1}{2} k+\frac{3}{4}\right)+\frac{(-1)^{k}}{4}
$$

which can be easily seen to agree with the result obtained from (A.18).

\section{Lifting the geometric action to an action on the bundle}

Before proceeding to more involved examples, let us describe an important subtlety that we have ignored in the previous examples, namely, the fact that a single geometric action can have multiple lifts to the complete line bundle. In our context, which lift we consider can be encoded in the way we describe the $\mathbb{Z}_{2}$ action. Consider for example $\mathbb{P}^{2}$, described above. We took the involution to be given by:

$$
\sigma:\left(x_{0}, x_{1}, x_{2}\right) \rightarrow\left(-x_{0}, x_{1}, x_{2}\right)
$$

but due to the $\mathbb{C}^{*}$ symmetry of $\mathbb{P}^{2}$, an equivalent description of this geometric action is

$$
\tau:\left(x_{0}, x_{1}, x_{2}\right) \rightarrow\left(x_{0},-x_{1},-x_{2}\right) .
$$


This ambiguity in fact encodes the two possible lifts of the geometric action on $\mathbb{P}^{2}$ to the bundle. Applying the same recipe as before, but now with $\tau$ instead of $\sigma$, we obtain that the sections of the bundle transform as:

$$
\tau^{*}: x_{0}^{a} x_{1}^{b} x_{2}^{k-a-b} \rightarrow(-1)^{k-a} x_{0}^{a} x_{1}^{b} x_{2}^{k-a-b} .
$$

That is, the action on each section gets multiplied by $(-1)^{k}$ with respect to (A.18). It is easy to check that with the prescription for the characters given above, the Lefschetz index reproduces this result: the fixed components $x_{0}=0, x_{1}=x_{2}=0$ now have characters $(-1)^{k}$ and 1 respectively, and thus:

$$
\chi^{\tau}\left(\mathbb{P}^{2}, \mathcal{O}(k)\right)=(-1)^{k} \chi^{\sigma}\left(\mathbb{P}^{2}, \mathcal{O}(k)\right) .
$$

From these arguments we see the need to define an action on the bundle in addition to the action on the geometry. In the mathematical literature this is commonly known as introducing an equivariant structure on the bundle. Let us briefly review this standard discussion.

Consider a vector bundle $V \stackrel{\pi}{\rightarrow} X$ and an action of a discrete group $G$ on $X$. If for each $g \in G$ there exists a bundle morphism $\phi_{g}: V \rightarrow V$ such that the diagram

$$
\begin{array}{cc}
V \stackrel{\phi_{g}}{\longrightarrow} V \\
\pi \downarrow \\
\quad & \downarrow \\
X \stackrel{g}{\longrightarrow} & X
\end{array}
$$

commutes for all $g \in G$, then the bundle morphisms define an invariant structure on $V$. If the morphisms also satisfy the relation

$$
\phi_{g} \circ \phi_{h}=\phi_{g h}
$$

for all $g, h \in G$, then the morphisms define an equivariant structure on $V$. An equivariant structure induces a map on sections. Requiring that the diagram

$$
\begin{array}{ccc}
V \stackrel{\phi_{g}}{\longrightarrow} & V \\
s \uparrow & \uparrow & s^{\prime} . \\
& X \stackrel{g}{\longrightarrow} X
\end{array}
$$

commutes for all $g \in G$ gives a map $\Phi_{g}: \Gamma(X, V) \rightarrow \Gamma(X, V)$ on sections defined by

$$
s^{\prime}=\Phi_{g}(s)=\phi_{g} \circ s \circ g^{-1} .
$$

Using the fact that $\phi_{g}$ give an equivariant structure, it can be shown that these maps obey the property $\Phi_{g} \circ \Phi_{h}=\Phi_{g h}$.

Having discussed equivariant structures and their induced sections, we would like to try to understand the above discussions in this formalism. For the purposes of this paper, the group $G$ is that of the holomorphic involution corresponding to the orientifold, so that $G=\mathbb{Z}_{2}$. In studying equivariant indices and cohomology, it is natural to study the induced 
(possibly local) section $\Phi_{g}(s)$ corresponding to a $g \in \mathbb{Z}_{2}$. Representing the $\mathbb{Z}_{2}$ action on $\mathbb{P}^{2}$ as $\mathbb{Z}_{2}=\{1, \sigma\}$, the morphisms

$$
\phi_{1}: p \times n \mapsto 1 \cdot p \times n \quad \phi_{\sigma}: p \times n \mapsto \sigma \cdot p \times n
$$

act on $p \times n \in \mathbb{P}^{2} \times \mathbb{C}$ and give an equivariant structure on $\mathcal{O}_{\mathbb{P}^{2}}(k)$. A generic section $s$ is of the form $s:\left(x_{0}, x_{1}, x_{2}\right) \mapsto\left(x_{0}, x_{1}, x_{2}\right) \times x_{0}^{a} x_{1}^{b} x_{2}^{k-a-b}$. A corresponding induced section is $s^{\prime}=\Phi_{\sigma}(s)=\phi_{\sigma} \circ s \circ \sigma^{-1}$ so that

$$
s^{\prime}:\left(x_{0}, x_{1}, x_{2}\right) \mapsto\left(x_{0}, x_{1}, x_{2}\right) \times(-1)^{a} x_{0}^{a} x_{1}^{b} x_{2}^{k-a-b} \equiv(-1)^{a} s,
$$

and we see that the equivariant structure we have defined gives the expected induced section. Furthermore, it is trivial that $\Phi_{1}(s)=\Phi_{\sigma} \circ \Phi_{\sigma}(s)=s$, and thus we have determined all induced sections under the $\mathbb{Z}_{2}$ equivariant structure. Similarly, if we represent the $\mathbb{Z}_{2}$ action on $\mathbb{P}^{2}$ by $\mathbb{Z}_{2}=\{1, \tau\}$, and define $\phi_{1}$ and $\phi_{\tau}$ similarly, one obtains $\Phi_{1}(s)=s$ and $\Phi_{\tau}(s)=(-1)^{k-a} s$, as obtained above.

The point of this discussion is that to study equivariant indices and equivariant line bundle cohomology, one must specify an equivariant structure in addition to the group action on the manifold. In the language of string theory, this means that an equivariant structure is part of the input data for a large volume type IIB orientifold compactification, in addition to the Calabi-Yau manifold and the holomorphic involution.

\section{Blow-up of $\mathbb{P}^{2}$ at one point}

Let us analyze a further example that tests the previous results in a slightly more non-trivial way: the blow-up of $\mathbb{P}^{2}$ at one point, also known as the first del Pezzo surface $d P_{1}$. We can represent this space torically by the gauged linear sigma model (GLSM) in table 6 .

\begin{tabular}{c|cccc} 
& $x_{1}$ & $x_{2}$ & $x_{3}$ & $x_{4}$ \\
\hline $\mathbb{C}_{1}^{*}$ & 1 & 1 & 1 & 0 \\
$\mathbb{C}_{2}^{*}$ & 0 & 0 & 1 & 1
\end{tabular}

Table 6: GLSM for $d P_{1}$.

There are two possible $\mathbb{Z}_{2}$ involutions that we can consider in this space [77]. Let us focus on the one given by

$$
\left(x_{1}, x_{2}, x_{3}, x_{4}\right) \rightarrow\left(-x_{1}, x_{2}, x_{3}, x_{4}\right) .
$$

We consider a line bundle $\mathcal{O}(m, n)$ of charge $(m, n)$ under $\mathbb{C}_{1,2}^{*}$, with $m \geq 0, n \geq 0$ for simplicity. The nonvanishing cohomology of this bundle comes only from $H^{i}\left(d P_{1}, \mathcal{O}(m, n)\right)$, with $i=0,1$. In particular, contributions to $H^{0}$ come from monomials $\prod x_{i}^{a_{i}}$ with $a_{i} \geq 0$ and total charge $(m, n)$ under $\mathbb{C}_{i}^{*}$, and contributions to $H^{1}$ come from rationoms [40] of the form:

$$
\mathfrak{h}=\frac{T\left(x_{3}, x_{4}\right)}{x_{1} x_{2} W\left(x_{1}, x_{2}\right)}
$$

such that $T$ and $W$ are monomials of positive degree of the respective variables, and the total charge of $\mathfrak{h}$ is $(m, n)$. As before, the action of $\sigma$ on the cohomology can be read simply from its action on the representative rationoms:

$$
\prod x_{i}^{a_{i}} \rightarrow(-1)^{a_{1}} \prod x_{i}^{a_{i}}, \quad \mathfrak{h} \rightarrow(-1)^{1+\operatorname{ord}_{1}(W)} \mathfrak{h},
$$

where $\operatorname{ord}_{1}(W)$ means the order $a$ of $x_{1}$ in $W\left(x_{1}, x_{2}\right)=x_{1}^{a} x_{2}^{b}$. 
The fixed point locus of $\sigma$ in this case is given by the curve $x_{1}=0$, and the two points $x_{2}=x_{3}=0, x_{2}=x_{4}=0$. The Lefschetz formula gives:

$$
\chi^{\sigma}\left(d P_{1}, \mathcal{O}(m, n)\right)=\frac{1}{2}(n+1)+\frac{(-1)^{m}}{4}+\frac{(-1)^{m+n}}{4},
$$

which can be seen to agree with the action on the rationoms.

\section{Another $\mathbb{Z}_{2}$ quotient of $d P_{1}$}

Let us study the other possible $\mathbb{Z}_{2}$ quotient of $d P_{1}$ :

$$
\sigma_{2}:\left(x_{1}, x_{2}, x_{3}, x_{4}\right) \rightarrow\left(x_{1}, x_{2}, x_{3},-x_{4}\right)
$$

The discussion for the action on the rationoms proceeds analogously to the previous case, with the resulting action being

$$
\prod x_{i}^{a_{i}} \rightarrow(-1)^{a_{4}} \prod x_{i}^{a_{i}}, \quad \mathfrak{h} \rightarrow(-1)^{1+\operatorname{ord}_{4}(T)} \mathfrak{h} .
$$

The fixed point locus consists of two disjoint curves: $x_{3}=0$ and $x_{4}=0$. The Lefschetz formula is in this case

$$
\chi^{\sigma_{2}}\left(d P_{1}, \mathcal{O}(m, n)\right)=\frac{1}{2}\left(m-n+\frac{1}{2}\right)+\frac{(-1)^{n}}{2}\left(m+\frac{3}{2}\right),
$$

which can easily be seen to give results consistent with the action on the cohomology induced from (A.35).

\section{Blow-up of $\mathbb{P}^{2}$ at three points}

Let us now consider the blow-up of $\mathbb{P}^{2}$ at three points, also known as the third del Pezzo surface $d P_{3}$. The GLSM model data for $d P_{3}$ is shown in table 7 .

Consider a bundle $\mathcal{L}$ with charges $q_{i}$ under the $\mathbb{C}_{i}^{*}$, and a $\mathbb{Z}_{2}$ action $\sigma$ given by:

$\sigma:\left(x_{1}, x_{2}, x_{3}, x_{4}, x_{5}, x_{6}\right) \rightarrow\left(x_{1}, x_{2}, x_{3}, x_{4}, x_{5},-x_{6}\right)$

This action has a couple of fixed curves at $x_{1}=$ 0 and $x_{6}=0$, with characteristic $(-1)^{q_{4}}$ and 1

\begin{tabular}{c|cccccc} 
& $x_{1}$ & $x_{2}$ & $x_{3}$ & $x_{4}$ & $x_{5}$ & $x_{6}$ \\
\hline $\mathbb{C}_{1}^{*}$ & 1 & 1 & 1 & 0 & 0 & 0 \\
$\mathbb{C}_{2}^{*}$ & 0 & 0 & 1 & 1 & 0 & 0 \\
$\mathbb{C}_{3}^{*}$ & 0 & 1 & 0 & 0 & 1 & 0 \\
$\mathbb{C}_{4}^{*}$ & 1 & 0 & 0 & 0 & 0 & 1
\end{tabular}

Table 7: GLSM charges for $d P_{3}$. respectively, and two fixed points at $x_{3}=x_{5}=0$ and $x_{2}=x_{4}=0$, with characteristics $(-1)^{q_{1}+q_{3}+q_{4}}$ and $(-1)^{q_{1}+q_{2}+q_{4}}$ respectively. ${ }^{17}$ From here, the equivariant Lefschetz index is easily calculated to be:

$$
\begin{aligned}
\chi^{\sigma}\left(d P_{3}, \mathcal{L}\right) & =\frac{1}{2}\left(q_{1}-q_{4}+\frac{1}{2}\right)+\frac{(-1)^{q_{4}}}{2}\left(-q_{1}+q_{2}+q_{3}+\frac{1}{2}\right) \\
& +\frac{(-1)^{q_{1}+q_{3}+q_{4}}}{4}+\frac{(-1)^{q_{1}+q_{2}+q_{4}}}{4} .
\end{aligned}
$$

\footnotetext{
${ }^{17}$ Looking at the GLSM only, one also obtains $x_{2}=x_{3}=0$ as a fixed locus, but it is in the Stanley-Reisner ideal of $d P_{3}$.
} 
In order to compute the action of the involution $\sigma$ on the cohomology we proceed as before, and assign to each element of the cohomology a sign given by the straight lift of the action (A.37) to the representative rationoms. In the case of $d P_{3}$ we encounter for the first time the issue of a given rationom contributing more than one element to the cohomology, encoded in the appearance of non-trivial remnant cohomology in the algorithm of [40]. By studying examples, we see that the whole secondary (remnant) cohomology transforms in the same way as the representative rationom, and thus one just needs to multiply by the appropriate prefactor when calculating equivariant indices. This agrees with the observation made at the beginning of this section that orientifolds acting as a sign change do not act on the Čech complex for a given local section.

\section{A realistic example}

The previous examples are illuminating but not particularly realistic. For our purposes we are interested in computing equivariant line bundle cohomology on four complex dimensional toric ambient spaces, rather than complex surfaces as we discussed above. Let us briefly discuss the issues that arise in applying the formalism above to a realistic example. ${ }^{18}$

Consider the $4 \mathrm{~d}$ toric variety $\mathcal{A}$ described by the GLSM data in table 8 . This space admits a smooth triangulation described by the Stanley-Reisner ideal:

$\mathcal{I}(\mathcal{A})=\left\langle x_{1} x_{3}, x_{1} x_{2} x_{5}, x_{3} x_{4} x_{6}, x_{4} x_{6} x_{7}, x_{2} x_{5} x_{7}\right\rangle$

\begin{tabular}{c|ccccccc} 
& $x_{1}$ & $x_{2}$ & $x_{3}$ & $x_{4}$ & $x_{5}$ & $x_{6}$ & $x_{7}$ \\
\hline $\mathbb{C}_{1}^{*}$ & 1 & 1 & 1 & 0 & 1 & 0 & 0 \\
$\mathbb{C}_{2}^{*}$ & 0 & 0 & 1 & 1 & 0 & 1 & 0 \\
$\mathbb{C}_{3}^{*}$ & 0 & 1 & 0 & 0 & 1 & 0 & 1
\end{tabular}

Table 8: GLSM data for $\mathcal{A}$.

We take the $\mathbb{Z}_{2}$ involution to be $\sigma: x_{4} \mapsto-x_{4}$. This obviously leaves the $x_{4}=0$ locus invariant, and by using $\mathbb{C}^{*}$ gauge transformations it is easy to see that the following loci are also fixed under the involution:

$$
\begin{aligned}
x_{3}=x_{6}=0 & & (-1)^{n} \\
x_{1}=x_{6}=x_{7}=0 & & (-1)^{n+m+p}
\end{aligned}
$$

We have listed next to each component of the fixed locus the character of a line bundle $\mathcal{L}=\mathcal{O}(m, n, p)$ on it, with $(m, n, p)$ denoting as usual the charges of the bundle under the $\mathbb{C}^{*}$ symmetries of the GLSM. There is formally another component of the fixed locus given by $x_{1}=x_{2}=x_{5}=x_{6}=0$, but it belongs to the Stanley-Reisner ideal and therefore we do not consider it further.

We see that in this case we have fixed loci of complex codimension 1, 2 and 3. Expanding (A.2) to the relevant orders, we get the following expressions. For the codimension 1 surface $D_{4}:\left\{x_{4}=0\right\}$, there is contribution to the index given by:

$$
\begin{aligned}
\delta \chi^{\sigma}(\mathcal{A}, \mathcal{O}(m, n, p))= & \frac{1}{2} \int_{D_{4}} \operatorname{ch}(\mathcal{O}(m, n, p)) \frac{\operatorname{Td}\left(T D_{4}\right)}{1-\frac{1}{2} D_{4}+14 D_{4}^{2}-\frac{1}{12} D_{4}^{3}+\ldots} \\
= & \frac{1}{48}\left(-4 m^{3}+12 m^{2} n-12 m n^{2}+4 n^{3}+12 m p^{2}-8 p^{3}\right. \\
& \left.\quad-6 m^{2}+12 m n-6 n^{2}+36 m p-12 p^{2}+28 m-4 n+20 p+27\right)
\end{aligned}
$$

\footnotetext{
${ }^{18}$ In dealing with realistic toric spaces we found the computer program SAGE [103] extremely useful, in particular in conjunction with its package for dealing with toric varieties [104].
} 
The contribution from the codimension 2 surface $\mathcal{S}:\left\{x_{3}=x_{6}=0\right\}$ is:

$$
\begin{aligned}
\delta \chi^{\sigma}(\mathcal{A}, \mathcal{O}(m, n, p)) & =\frac{(-1)^{n}}{4} \int_{\mathcal{S}} \operatorname{ch}(\mathcal{O}(m, n, p)) \frac{\operatorname{Td}(T \mathcal{S})}{1-\operatorname{ch}\left(\overline{D_{3}} \oplus \overline{D_{6}}\right)+\operatorname{ch}\left(\overline{D_{3}} \otimes \overline{D 6}\right)} \\
& =\frac{(-1)^{n}}{8}\left(p^{2}+3 p+2\right)
\end{aligned}
$$

where we have denoted by $D_{3}$ and $D_{6}$ the line bundles associated to the corresponding divisors. We also have a fixed curve $\mathcal{C}:\left\{x_{1}=x_{6}=x_{7}=0\right\}$, which gives a contribution:

$$
\begin{aligned}
\delta \chi^{\sigma}(\mathcal{A}, \mathcal{O}(m, n, p)) & =\frac{(-1)^{n+m+p}}{8} \int_{\mathcal{C}} \operatorname{ch}(\mathcal{O}(m, n, p)) \frac{\operatorname{Td}(T \mathcal{C})}{1-\frac{1}{8}\left(\left[D_{1}\right]+\left[D_{6}\right]+\left[D_{7}\right]\right)} \\
& =\frac{(-1)^{n+m+p}}{48}(6 p+9)
\end{aligned}
$$

Finally, and although we have no such case in our example, let us mention for completeness that fixed points would give a contribution of $\pm 1 / 16$ to the Lefschetz index. The Lefschetz equivariant index in our example is thus the sum of (A.41), (A.42) and (A.43).

Let us check in a simple example that the result of our prescription on the rationoms agrees with the Lefschetz index. Consider the line bundle $\mathcal{O}(1,1,1)$. For this bundle only $H^{0}$ is non-vanishing. The contributing sections with their sign under $\sigma: x_{4} \mapsto-x_{4}$ are:

$\begin{array}{cccc}x_{3} x_{7} & (+) & x_{1} x_{6} x_{7} & (+) \\ x_{1} x_{4} x_{7} & (-) & x_{5} x_{6} & (+) \\ x_{2} x_{6} & (+) & x_{4} x_{5} & (-) \\ x_{2} x_{4} & (-) & & \end{array}$

From here, it is clear that $h_{+}^{0}-h_{-}^{0}=1$. Substituting $m=n=p=1$ in the expression for the Lefschetz equivariant index found above agrees with this result.

\section{A.3 Koszul resolution}

In the previous section we have discussed how to compute equivariant line bundle cohomology on toric varieties. Nevertheless, in physical applications one is actually interested in equivariant line bundle cohomology on varieties which are not toric, compact Calabi-Yau spaces being a notable example. Luckily, most spaces of interest (denoted $X$ in what follows) can be embedded as complete intersections of hypersurfaces in toric ambient spaces $\mathcal{A}$, and one can carry over the information obtained in the previous section to the subspace.

Our basic tool will be the Koszul complex:

$$
0 \rightarrow N^{*} \stackrel{f}{\rightarrow} \mathcal{O}_{\mathcal{A}} \stackrel{r}{\rightarrow} \mathcal{O}_{X} \rightarrow 0
$$

where $N^{*}=\mathcal{O}_{\mathcal{A}}(-X)$ is the dual to the normal bundle of $X$ in $\mathcal{A}$, and we are assuming here for ease of exposition that $X$ is a divisor in $\mathcal{A}$ (the general expression is given for example in the appendix of [39]). The first map is multiplication by the section $f=0$ defining $X$, and the second map is restriction to $X$. For our applications, it will be convenient to tensor this exact sequence with appropriate line bundles $\mathcal{O}(D)$, giving:

$$
0 \rightarrow \mathcal{O}_{\mathcal{A}}(D-X) \stackrel{f}{\rightarrow} \mathcal{O}_{\mathcal{A}}(D) \stackrel{r}{\rightarrow} \mathcal{O}_{X}(D) \rightarrow 0
$$


By the snake lemma, this exact sequence gives a long exact sequence in cohomology:

$$
\begin{aligned}
0 & \rightarrow H^{0}\left(\mathcal{A}, \mathcal{O}_{\mathcal{A}}(D-X)\right) \rightarrow H^{0}\left(\mathcal{A}, \mathcal{O}_{\mathcal{A}}(D)\right) \rightarrow H^{0}\left(X, \mathcal{O}_{X}(D)\right) \rightarrow \\
& \rightarrow H^{1}\left(\mathcal{A}, \mathcal{O}_{\mathcal{A}}(D-X)\right) \rightarrow H^{1}\left(\mathcal{A}, \mathcal{O}_{\mathcal{A}}(D)\right) \rightarrow H^{1}\left(X, \mathcal{O}_{X}(D)\right) \rightarrow \ldots
\end{aligned}
$$

where the maps are the ones induced from (A.46). As with any long exact sequence of abelian groups, this exact sequence can be split into short exact sequences. In general, given a long exact sequences of abelian groups:

$$
\ldots \rightarrow A \rightarrow B \rightarrow C \rightarrow D \rightarrow E \rightarrow 0
$$

there exists a $X$ such that both

$$
\ldots \rightarrow A \rightarrow B \rightarrow C \rightarrow X \rightarrow 0
$$

and

$$
0 \rightarrow X \stackrel{x}{\rightarrow} D \rightarrow E \rightarrow 0
$$

are exact. In this way we can split any long exact sequence such as (A.47) into short exact sequences. Consider for example the short exact sequence (A.50). We have that

$$
E \simeq \frac{D}{\operatorname{Im}(X)},
$$

where $\operatorname{Im}(X)$ denotes the image of $X$ under $x$. Since $x$ is injective, $\operatorname{Im}(X)$ can be thought of as an embedding of $X$ in $D$.

Consider now the case of the Koszul sequence (A.47), and for simplicity let us just assume that $H^{1}\left(\mathcal{A}, \mathcal{O}_{\mathcal{A}}(X-D)\right)=0$. Therefore we do not have to split the long exact sequence, since we already have the short exact sequence

$$
0 \rightarrow H^{0}\left(\mathcal{A}, \mathcal{O}_{\mathcal{A}}(D-X)\right) \stackrel{f}{\rightarrow} H^{0}\left(\mathcal{A}, \mathcal{O}_{\mathcal{A}}(D)\right) \rightarrow H^{0}\left(X, \mathcal{O}_{X}(D)\right) \rightarrow 0 .
$$

and thus we have that

$$
H^{0}\left(X, \mathcal{O}_{X}(D)\right) \simeq \frac{H^{0}\left(\mathcal{A}, \mathcal{O}_{\mathcal{A}}(D-X)\right)}{f^{*}\left(H^{0}\left(\mathcal{A}, \mathcal{O}_{\mathcal{A}}(D)\right)\right)}
$$

Recall that the map $f$ denotes multiplication by the equation defining the hypersurface $X$, and here $f^{*}$ is its pullback to the space of sections (we can also think of this as multiplying by an specific section of $\left.\mathcal{O}_{\mathcal{A}}(D)\right)$. Notice that we have obtained that the unknown cohomology group $H^{0}\left(X, \mathcal{O}_{X}(D)\right)$ can be expressed in terms of known cohomologies in the ambient space. In order to study systematically the representation of $H^{0}\left(X, \mathcal{O}_{X}(D)\right)$ under the $\mathbb{Z}_{2}$ action, it will be convenient to introduce some elementary group theory.

Elements of line bundle cohomology groups transform in specific representations of the $\mathbb{Z}_{2}$ action, and we would now want to split into irreps of $\mathbb{Z}_{2}$, namely terms that transform with a plus sign and terms that transform with a minus sign. Since $\mathbb{Z}_{2}$ is a finite group, this information is completely encoded in the group character:

$$
\chi_{g}(R)=\operatorname{Tr}_{R}(g)
$$


where $\operatorname{Tr}_{R}$ simply denotes the trace over the representation $R$. By Schur orthogonality, the characters of the irreps of a finite group are orthogonal under the inner product

$$
\chi(R) \cdot \chi(S)=\frac{1}{|G|} \sum_{g} \chi_{g}^{*}(R) \chi_{g}(S)
$$

where $\chi(R)=\left(\chi_{1}(R), \ldots, \chi_{g_{|G|}}(R)\right)$ and $|G|$ is the order of the group $\left(2\right.$ for $\left.\mathbb{Z}_{2}\right)$. This is evident in our case, since

$$
\chi_{g}(+)=(1,1), \quad \chi_{g}(-)=(1,-1) .
$$

where we have denoted by \pm the trivial and fundamental representations of $\mathbb{Z}_{2}$. From these expressions, if we have a cohomology group $H$ decomposing as $H_{+} \oplus H_{-}$, we have that

$$
\operatorname{dim} H_{ \pm}=\frac{1}{2} \chi(H) \cdot(1, \pm 1) .
$$

So in order to obtain the relevant dimensions, we need to know the character of $H$.

Let us come back to (A.53). Consider first the case in which the map $f$ is invariant under the $\mathbb{Z}_{2}$ involution. In this case, by the isomorphism (A.53) and some basic facts about representation theory [105] (see also [106] for a similar recent discussion in the context of the heterotic string) we have that:

$$
\chi_{g}\left(H^{0}(X, \mathcal{O}(D))\right)=\chi_{g}\left(H^{0}\left(\mathcal{A}, \mathcal{O}_{\mathcal{A}}(D)\right)\right)-\chi_{g}\left(H^{0}\left(\mathcal{A}, \mathcal{O}_{\mathcal{A}}(X-D)\right)\right) .
$$

The other possibility is that $f$ takes a minus sign when we act with $\mathbb{Z}_{2}(f=0$ is still invariant, of course). In this case the inclusion map $f^{*}$ introduces an additional minus sign into (A.58), which is now given by:

$$
\chi_{g}\left(H^{0}(X, \mathcal{O}(D))\right)=\chi_{g}\left(H^{0}\left(\mathcal{A}, \mathcal{O}_{\mathcal{A}}(D)\right)\right)-g \cdot \chi_{g}\left(\left(H^{0}\left(\mathcal{A}, \mathcal{O}_{\mathcal{A}}(X-D)\right)\right)\right) .
$$

where $g= \pm 1$. A similar discussion applies for more complicated situations, with the net effect that one has to multiply $H^{i}\left(\mathcal{A}, \mathcal{O}_{\mathcal{A}}(X-D)\right)$ by $g$ when using character addition formulas such as (A.59).

Example: $d P_{1} \subset \mathbb{P}^{2} \times \mathbb{P}^{1}$

As an illustration of the method, let us discuss line bundle cohomology for $d P_{1}$, now understood as a hypersurface in $\mathbb{P}^{2} \times \mathbb{P}^{1}$. In particular, denoting as $\left(z_{0}, z_{1}, z_{2} \mid y_{0}, y_{1}\right)$ the coordinates of $\mathbb{P}^{2} \times \mathbb{P}^{1}, d P_{1}$ can be understood as any hypersurface of the form $\sum c_{i k} z_{i} y_{k}=0$. For definiteness, let us take $f=z_{1} y_{1}+z_{2} y_{0}=0$ to be our chosen representative. Since we will want to compare results with the results derived above for the equivariant cohomology of $d P_{1}$, let us parameterize the $d P_{1}$ as above in table 6 , in terms of $x_{1} \ldots x_{4}$. In these variables, it is easy to see that one explicit embedding is given by:

$$
\begin{aligned}
z_{0}=x_{3} & y_{0}=x_{2} \\
z_{1}=x_{2} x_{4} & y_{1}=-x_{1} \\
z_{2}=x_{1} x_{4} &
\end{aligned}
$$


Consider a $\mathbb{Z}_{2}$ action on $\mathbb{P}^{2} \times \mathbb{P}^{1}$ of the form: ${ }^{19}$

$$
\left(z_{0}, z_{1}, z_{2} \mid y_{0}, y_{1}\right) \rightarrow\left(z_{0}, z_{1},-z_{2} \mid-y_{0}, y_{1}\right)
$$

This action leaves fixed a curve and two points inside the $d P_{1}$ hypersurface, and we can thus identify it with the first involution of $d P_{1}$ studied above. Indeed, from the embedding (A.60) we obtain an induced action on the $d P_{1}$ sections:

$$
\left(x_{1}, x_{2}, x_{3}, x_{4}\right) \rightarrow\left(x_{1},-x_{2}, x_{3},-x_{4}\right) .
$$

As a further piece of information before going into the Koszul resolution, we need to identify the divisors in the ambient space with the divisors in the $d P_{1}$ hypersurface. This is easily done by imposing that the induced intersection forms and the tangent bundle on $d P_{1}$ agree in both bases. Denoting $D_{1}, D_{2}$ the hyperplanes of $\mathbb{P}^{1}$ and $\mathbb{P}^{2}$ respectively, and $H, X$ the $(1,0)$ and $(0,1)$ divisors of $d P_{1}$ in the conventions of the previous section, we have that $X=D_{2}-D_{1}, H=D_{1}$.

From here on it is just a matter of checking the formulas. Take for example the divisor $\mathcal{O}_{d P 1}(3 H+X)$. The only non-vanishing elements of the induced cohomology are (we abbreviate $\mathbb{P}^{2} \times \mathbb{P}^{1}$ to $\mathcal{A}$ ):

$$
0 \rightarrow H^{0}\left(\mathcal{A}, \mathcal{O}_{\mathcal{A}}\left(D_{1}\right)\right) \rightarrow H^{0}\left(\mathcal{A}, \mathcal{O}_{\mathcal{A}}\left(D_{2}+2 D_{1}\right)\right) \rightarrow H^{0}\left(d P_{1}, \mathcal{O}_{d P 1}(3 H+X)\right) \rightarrow 0 .
$$

An easy computation using the techniques described above, or alternatively a combination of more classical methods such as the Künneth formula and Lefschetz's equivariant index theorem, gives:

$$
\begin{aligned}
\chi_{g}\left(H^{0}\left(\mathcal{A}, \mathcal{O}_{\mathcal{A}}\left(D_{1}\right)\right)\right) & =(2,0) \\
\chi_{g}\left(H^{0}\left(\mathcal{A}, \mathcal{O}_{\mathcal{A}}\left(2 D_{1}+D_{2}\right)\right)\right) & =(9,1)
\end{aligned}
$$

We have that $f=z_{1} y_{1}+z_{2} y_{0}$ is invariant under (A.61), so using (A.58) we deduce that:

$$
\chi_{g}\left(H^{0}\left(d P_{1}, \mathcal{O}_{d P 1}(3 H+X)\right)=(7,1)\right.
$$

which can be easily verified independently using the formulas in the previous section. Notice that it is important when checking these formulas to take the proper restriction of the bundle $\mathbb{Z}_{2}$ action, this is the one given in (A.62).

As another quick example, consider the $\mathbb{Z}_{2}$ action leaving two curves on $d P_{1}$ fixed:

$$
\left(x_{1}, x_{2}, x_{3}, x_{4}\right) \rightarrow\left(x_{1}, x_{2}, x_{3},-x_{4}\right) .
$$

It is easy to see that this lifts to:

$$
\left(z_{0}, z_{1}, z_{2} \mid y_{0}, y_{1}\right) \rightarrow\left(z_{0},-z_{1},-z_{2} \mid y_{0}, y_{1}\right)
$$

Taking the same bundle as before, the characters are now:

$$
\begin{aligned}
\chi_{g}\left(H^{0}\left(\mathcal{A}, \mathcal{O}_{\mathcal{A}}\left(D_{1}\right)\right)\right) & =(2,2) \\
\chi_{g}\left(H^{0}\left(\mathcal{A}, \mathcal{O}_{\mathcal{A}}\left(2 D_{1}+D_{2}\right)\right)\right) & =(9,-3)
\end{aligned}
$$

\footnotetext{
${ }^{19}$ Recall that in our conventions (A.61) actually specifies an action on the bundle, not just on the geometry.
} 
We now have that $f=z_{1} y_{1}+z_{2} y_{0}$ changes sign under the $\mathbb{Z}_{2}$ action (A.67), and thus using (A.59) we have that:

$$
\chi_{g}\left(H^{0}\left(d P_{1}, \mathcal{O}_{d P 1}(3 H+X)\right)=(7,-1) .\right.
$$

which can also be checked independently using the techniques of the previous section.

\section{A.4 Permutation orientifolds}

So far we have dealt with $\mathbb{Z}_{2}$ involutions that act by at most a sign on the GLSM coordinates. This is enough for treating the examples in the main text, but it is not the most general class of possible $\mathbb{Z}_{2}$ involutions one may consider. In this section we would like to briefly discuss the extension of the ideas of the previous section to involution exchanging GLSM coordinates. Namely, we allow actions of the form:

$$
\pi_{2}:\left(x_{1}, x_{2}, \ldots, x_{n}\right) \mapsto\left(x_{p_{2}(1)}, x_{p_{2}(2)}, \ldots, x_{p_{2}(n)}\right)
$$

where $p_{2}$ is an order 2 permutation of $\{1,2, \ldots, n\}$. Such $\mathbb{Z}_{2}$ involutions have been considered recently in the context of F-theory model building [63, 64].

There are a few interesting subtleties that appear in this case. Since now the permutation acts on the coordinates, it can act non-trivially on the Čech complex we obtain out of each local section. In principle, in order to compute equivariant line bundle cohomology in this case we would need to define this action on the Cech complex carefully, and obtain in this way the induced representation on the Čech cohomology for each chamber. ${ }^{20}$ There is nevertheless a simple shortcut we can use in order to avoid having to do this. Notice that, since each local section in the same Čech chamber gives rise to the same Čech complex, for each chamber we have a direct product structure for the representation of the $\mathbb{Z}_{2}$ action. Namely, when computing the character of $\pi_{2}$ on a particular chamber $C$, we can write:

$$
\operatorname{Tr}_{C}\left(\pi_{2}\right)=\operatorname{Tr}_{\check{c}}\left(\pi_{2}\right) \cdot \operatorname{Tr}_{M}\left(\pi_{2}\right)
$$

where $\check{c}$ is the cohomology group coming from each section in the chamber, and $M$ is the space of sections in the chamber. The second term $\operatorname{Tr}_{M}\left(\pi_{2}\right)$ denotes the trace of the $\mathbb{Z}_{2}$ action on the space of local sections in the chamber under consideration. Notice that we only need to consider chambers mapped to themselves under the $\mathbb{Z}_{2}$ action. If a chamber is not invariant the induced representation on the local sections always acts as an exchange of sections, i.e. a matrix of the form:

$$
M=\left(\begin{array}{cc}
0 & \pm 1 \\
\pm 1 & 0
\end{array}\right)
$$

which has zero trace, and thus does not contribute to the equivariant index.

Since we are taking $\operatorname{Tr}_{\check{c}}\left(\pi_{2}\right)$ to depend just on the structure of the Čech complex in the chamber, we can use index formulas to determine it in a few simple cases, and then use

\footnotetext{
${ }^{20}$ Here we are using ideas and terminology from the chamber algorithm for computing line bundle cohomology. We refer the reader to [102, 40, 39] for reviews of the relevant concepts.
} 
this for obtaining the general result. Let us illustrate how this works in a simple example. Consider the third del Pezzo surface $d P_{3}$, described by the GLSM data in table 7 above, and take the involution given by:

$$
\pi_{2}:\left(x_{1}, x_{2}, x_{3}, x_{4}, x_{5}, x_{6}\right) \mapsto\left(x_{2}, x_{1}, x_{3}, x_{4}, x_{6}, x_{5}\right) .
$$

We are interested in computing line bundle cohomology for an equivariant bundle of the form $\mathcal{O}(m, n, p, p)$. We have chosen this particular form in order for the line bundle to map to itself under the involution $\pi_{2}$. It is a simple calculation to show that the fixed point locus of (A.73) is given by the curve $x_{1} x_{5}-x_{2} x_{6}=0$ and the two points $\left\{x_{1} x_{5}+x_{2} x_{6}=0, x_{4}=0\right\},\left\{x_{1} x_{5}+x_{2} x_{6}=0, x_{3}=0\right\}$. The resulting Lefschetz equivariant index is then given by:

$$
\chi^{\pi_{2}}\left(d P_{3}, \mathcal{O}(m, n, p, p)\right)=\frac{1}{2}(n+1)+\frac{(-1)^{m}}{4}\left(1+(-1)^{n}\right) .
$$

As an example, consider the line bundle $\mathcal{O}(-2,0,0,0)$. The only non-vanishing contribution from a symmetric chamber comes from an element in the chamber $\left(x_{1} x_{2}\right)^{-1}$, understood as a term in the power-set of the Stanley-Reisner ideal [40, 41, 42], and in particular from the single local section $x_{5} x_{6} / x_{1} x_{2}$. In the notation of (A.71) we thus have that $\operatorname{Tr}_{M}\left(\pi_{2}\right)=1$, and since this is a contribution to $H^{1}\left(d P_{3}, \mathcal{O}(-2,0,0,0)\right)$, we have that:

$$
\chi^{\pi_{2}}\left(d P_{3}, \mathcal{O}(-2,0,0,0)\right)=-\operatorname{Tr}_{\check{c}}\left(\pi_{2}\right) \cdot 1=-\operatorname{Tr}_{\check{c}}\left(\pi_{2}\right)
$$

with $\check{c}$ being in this case the $\left(x_{1} x_{2}\right)^{-1}$ chamber. We can now compute the equivariant index independently using formula (A.74), and from there we obtain:

$$
\operatorname{Tr}_{\check{c}}\left(\pi_{2}\right)=-1 \text {. }
$$

The trace over the other contributing chambers can then be computed similarly. Knowledge of the result for all contributing chambers in the problem then allows us to compute equivariant line bundle cohomology for any line bundle using (A.71). For instance, the character $\chi_{\pi_{2}}\left(H^{1}\left(d P_{3}, \mathcal{O}(-2,2,0,0)\right)\right)$ receives contributions only the trace over the $\left(x_{1} x_{2}\right)^{-1}$ chamber. The space of contributing local sections in this chamber is generated by:

$$
\begin{array}{ccc}
\frac{x_{4}^{2} x_{5} x_{6}}{x_{1} x_{2}} & \frac{x_{3} x_{4} x_{5}^{2} x_{6}}{x_{1} x_{2}^{2}} & \frac{x_{3}^{2} x_{5}^{3} x_{6}}{x_{2} x_{2}^{3}} \\
\frac{x_{3} x_{4} x_{5} x_{6}^{2}}{x_{1}^{2} x_{2}} & \frac{x_{3}^{2} x_{5}^{6} x_{6}^{2}}{x_{1}^{2} x_{2}^{2}} & \frac{x_{3}^{2} x_{5} x_{6}^{3}}{x_{1}^{3} x_{2}}
\end{array}
$$

From here we find that $\operatorname{Tr}_{M}\left(\pi_{2}\right)=2$ (since there are 2 invariant local sections), and using (A.76) we then find that:

$$
\chi_{\pi_{2}}\left(H^{1}\left(d P_{3}, \mathcal{O}(-2,2,0,0)\right)\right)=-2 .
$$

As a simple check, this result agrees with the one obtained from the Lefschetz index (A.74). Since $\operatorname{dim}\left(H^{1}\left(d P_{3}, \mathcal{O}(-2,2,0,0)\right)\right)=8$, we have that

$$
\begin{aligned}
& h_{+}^{1}\left(d P_{3}, \mathcal{O}(-2,2,0,0)\right)=3 \\
& h_{-}^{1}\left(d P_{3}, \mathcal{O}(-2,2,0,0)\right)=5 .
\end{aligned}
$$




\section{B. Factorization: a geometric viewpoint}

In section 4, arguments for the factorization of intersection forms on certain manifolds were presented from an algebraic viewpoint. Specifically, given the Stanley-Reisner ideal of a $d$-dimensional ambient space toric variety $\mathcal{A}$ along with the data encoding linear equivalence, one can calculate the intersection ring explicitly to determine whether or not it factorizes. In the case where generators of the Stanley-Reisner ideal of some $k$-dimensional toric subvariety $\mathcal{B} \subset \mathcal{A}$ are also generators of the Stanley-Reisner ideal of $\mathcal{A}$ and linear equivalence of divisors in $\mathcal{B}$ is preserved in $\mathcal{A}$, then divisors in $\mathcal{B}$ pulled back to the ambient space can appear at most $k$ times in non-vanishing monomials in the intersection $d$-form of $\mathcal{A}$.

While the algebraic viewpoint makes everything explicit, one of the well-known virtues of toric varieties is that they are amenable to powerful combinatorial methods of analysis. For example, one set of lattice data which can be used to define a toric variety is a complete fan, which is a set of strongly convex rational polyhedral cones, each of which corresponds to an affine patch on the toric variety, along with some consistency conditions which the cones must satisfy. Each divisor corresponds to a ray in the lattice, and a set of divisors $\left\{D_{i_{1}}, \ldots, D_{i_{k}}\right\}$ (up to linear equivalence) can simultaneously vanish if and only if the corresponding rays are in some cone in the fan. If there is no such cone, this is the combinatorial description of the statement that $x_{i_{1}} \ldots x_{i_{k}}$ is in the Stanley-Reisner ideal.

Though we worked primarily with GLSM and algebraic data for discussions in the main text, in this appendix we would like to discuss briefly the combinatorial viewpoint on manifolds whose intersection form factorizes. Those readers familiar with toric geometry can probably already anticipate the answer: since the elliptic Calabi-Yau manifolds in the main text were constructed in a toric ambient space $\mathcal{A}$ by essentially taking the GLSM data corresponding to some $d$-dimensional toric variety $\mathcal{B}$ and augmenting it with a copy of $\mathbb{P}_{231}$, it is possible to see explicitly both the $d$-dimensional lattice data of $\mathcal{B}$ and the two-dimensional lattice data of $\mathbb{P}_{231}$ reflected in the $(d+2)$-dimensional lattice data of $\mathcal{A}$.

Before going into the general discussion, let us present an example to motivate it. We use as our example the GLSM data given in table 3 for a toric variety whose Calabi-Yau hypersurface is an elliptic fibration over the Hirzebruch surface $\mathbb{F}_{n}$. The point matrix

$$
v_{M N}=\left(\begin{array}{cccc|ccc}
1 & -1 & 0 & 0 & 0 & 0 & 0 \\
0 & -n & 1 & -1 & 0 & 0 & 0 \\
\hline-2 & -2 & -2 & -2 & 1 & 0 & -2 \\
-3 & -3 & -3 & -3 & 0 & 1 & -3
\end{array}\right)
$$

satisfies the relation $v_{M N} Q_{N}^{a}=0 \forall a$, as required by the theory of toric varieties, and the $Q^{a}$ vectors are just $M, N, O, P$ in the GLSM data. It is straightforward to check that this is indeed a solution, where the columns give the points in the four-dimensional $N$ lattice corresponding to $s, t, u, v, x, y$ and $z$, respectively. Moreover, the lattice data corresponding to both $\mathbb{F}_{n}$ and $\mathbb{P}_{231}$ are present in the upper left quadrant and the lower right quadrant, respectively. In fact, it can be seen by working out the linear algebra that this solution 
does not depend explicitly on the fan data corresponding to $\mathcal{B}$, but instead just on the fact that the point matrix of $\mathcal{B}$ annihilates the subset of the GLSM data associated with $\mathcal{B}$.

This fact suggests a generalization of the solution for $\mathcal{B}=\mathbb{F}_{n}$. Let $\mathcal{B}$ be a $d$-dimensional toric variety with $k$ homogeneous coordinates, which therefore has $(k-d)$ GLSM relations. Taking $A$ to be the $(d \times k)$ point matrix of $\mathcal{B}, B$ and $C$ to be precisely the lower left and lower right quadrants in the $\mathbb{F}_{n}$ solution, and $\mathbf{0}$ to be a $(d \times 3)$ matrix of zeroes, then

$$
v_{M N}=\left(\begin{array}{l|l}
A & \mathbf{0} \\
\hline B & C
\end{array}\right)
$$

is the point matrix of a $(d+2)$-dimensional toric variety with the point matrices of $\mathcal{B}$ and $\mathbb{P}_{231}$ explicit in the upper left and lower right quadrants. It has $(k+3)$ homogeneous coordinates and $(k-d+1)$ relations given by

$$
\tilde{Q}^{a}=\left(Q_{i}^{a}, 2 \sum_{i} Q_{i}^{a}, 3 \sum_{i} Q_{i}^{a}, 0\right) \quad \text { and } \quad \tilde{Q}^{a+1}=(\overbrace{0, \ldots, 0,2}^{\mathrm{k} \text { times }}, 3,1),
$$

where $Q_{i}^{a}$ are the $k$-dimensional charge vectors of $\mathcal{B}$. The fact that the point matrix annihilates these charge vectors relies heavily on the fact that the Calabi-Yau condition determined the last three components of $\tilde{Q}^{a}$. All $\mathbb{P}_{231}$ fibrations in the main text have GLSM data of this form.

From this example and our general solution, we see that the $k$-dimensional subspace $N_{\mathcal{B}}$ where the lattice data of $\mathcal{B}$ lives is $\left(x_{1}, \ldots, x_{k},-2,-3\right) \in \mathbb{R}^{d+2}$ and the 2-dimensional subspace $N_{231}$ where the lattice data of $\mathbb{P}_{231}$ lives is $\left(0, \ldots, 0, x_{d+1}, x_{d+2}\right) \in \mathbb{R}^{d+2}$. An interesting observation from the Hirzebruch example and also from the general solution is that the point corresponding to the $z$ coordinate is precisely at the origin of $N_{\mathcal{B}}$, i.e. at $(0, \ldots, 0,-2,-3)$. If $\mathcal{B}$ is a toric variety defined by a $*$-triangulation of a polytope $B \subset N_{\mathcal{B}}$ (whose origin is the $z$ point in the $(k+2$ )-dimensional lattice $N$ ), then every simplex in the triangulation necessarily contains the $z$ point. Since linear equivalence of divisors in $\mathcal{B}$ is preserved amongst their pullbacks in $\mathcal{A}$, any non-zero intersection of $k+1$ divisors in $\mathcal{A}$ whose points are in $N_{\mathcal{B}}$ must necessarily involve $D_{z}$. This is equivalent to factorization of the intersection $(k+2)$-form on $\mathcal{A}$, given our assumption above that the $\operatorname{Div}(\mathcal{A})$ generator corresponding to $\tilde{Q}^{a+1}$ is the only one which is not also a generator of $\operatorname{Div}(\mathcal{B})$.

\section{References}

[1] F. Denef, M. R. Douglas, and B. Florea, Building a better racetrack, JHEP 06 (2004) 034, [hep-th/0404257].

[2] V. Balasubramanian, P. Berglund, J. P. Conlon, and F. Quevedo, Systematics of Moduli Stabilisation in Calabi-Yau Flux Compactifications, JHEP 03 (2005) 007, [hep-th/0502058].

[3] F. Denef, M. R. Douglas, B. Florea, A. Grassi, and S. Kachru, Fixing all moduli in a simple F-theory compactification, Adv. Theor. Math. Phys. 9 (2005) 861-929, [hep-th/0503124].

[4] R. Blumenhagen, M. Cvetič, and T. Weigand, Spacetime instanton corrections in $4 D$ string vacua - the seesaw mechanism for D-brane models, Nucl. Phys. B771 (2007) 113-142, [hep-th/0609191]. 
[5] L. E. Ibanez and A. M. Uranga, Neutrino Majorana masses from string theory instanton effects, JHEP 03 (2007) 052, [hep-th/0609213].

[6] B. Florea, S. Kachru, J. McGreevy, and N. Saulina, Stringy Instantons and Quiver Gauge Theories, JHEP 05 (2007) 024, [hep-th/0610003].

[7] R. Blumenhagen, S. Moster, and E. Plauschinn, Moduli Stabilisation versus Chirality for MSSM like Type IIB Orientifolds, JHEP 01 (2008) 058, [arXiv:0711.3389].

[8] R. Blumenhagen, M. Cvetič, S. Kachru, and T. Weigand, D-Brane Instantons in Type II Orientifolds, Ann. Rev. Nucl. Part. Sci. 59 (2009) 269-296, [arXiv:0902.3251].

[9] N. Seiberg and E. Witten, Monopole Condensation, And Confinement In N=2 Supersymmetric Yang-Mills Theory, Nucl. Phys. B426 (1994) 19-52, [hep-th/9407087].

[10] N. Seiberg and E. Witten, Monopoles, duality and chiral symmetry breaking in N=2 supersymmetric QCD, Nucl. Phys. B431 (1994) 484-550, [hep-th/9408099].

[11] N. A. Nekrasov, Seiberg-Witten Prepotential From Instanton Counting, Adv. Theor. Math. Phys. 7 (2004) 831-864, [hep-th/0206161].

[12] R. Dijkgraaf and C. Vafa, Matrix models, topological strings, and supersymmetric gauge theories, Nucl. Phys. B644 (2002) 3-20, [hep-th/0206255].

[13] R. Dijkgraaf and C. Vafa, On geometry and matrix models, Nucl. Phys. B644 (2002) 21-39, [hep-th/0207106].

[14] R. Dijkgraaf and C. Vafa, A perturbative window into non-perturbative physics, hep-th/0208048.

[15] M. Aganagic, C. Beem, and S. Kachru, Geometric Transitions and Dynamical SUSY Breaking, Nucl. Phys. B796 (2008) 1-24, [arXiv:0709.4277].

[16] I. Garcia-Etxebarria, D-brane instantons and matrix models, JHEP 07 (2009) 017, [arXiv:0810.1482].

[17] A. Collinucci, P. Soler, and A. M. Uranga, Non-perturbative effects and wall-crossing from topological strings, JHEP 11 (2009) 025, [arXiv:0904.1133].

[18] C. Petersson, P. Soler, and A. M. Uranga, D-instanton and polyinstanton effects from type I' D0- brane loops, JHEP 06 (2010) 089, [arXiv:1001.3390].

[19] C. Vafa, Superstrings and topological strings at large N, J. Math. Phys. 42 (2001) 2798-2817, [hep-th/0008142].

[20] A. Lawrence and J. McGreevy, Local string models of soft supersymmetry breaking, JHEP 06 (2004) 007, [hep-th/0401034].

[21] M. Davidse, F. Saueressig, U. Theis, and S. Vandoren, Membrane instantons and de Sitter vacua, JHEP 09 (2005) 065, [hep-th/0506097].

[22] F. Saueressig, U. Theis, and S. Vandoren, On de Sitter vacua in type IIA orientifold compactifications, Phys. Lett. B633 (2006) 125-128, [hep-th/0506181].

[23] S. Alexandrov, D. Persson, and B. Pioline, On the topology of the hypermultiplet moduli space in type II/CY string vacua, arXiv:1009.3026.

[24] P. G. Camara and E. Dudas, Multi-instanton and string loop corrections in toroidal orbifold models, JHEP 08 (2008) 069, [arXiv:0806.3102]. 
[25] T. W. Grimm, Non-Perturbative Corrections and Modularity in N=1 Type IIB Compactifications, JHEP 10 (2007) 004, [arXiv:0705.3253].

[26] R. Blumenhagen, A. Collinucci, and B. Jurke, On Instanton Effects in F-theory, arXiv:1002.1894.

[27] I. Garcia-Etxebarria, F. Marchesano, and A. M. Uranga, Non-perturbative F-terms across lines of BPS stability, JHEP 07 (2008) 028, [arXiv:0805.0713].

[28] M. Billo' et. al., Flux interactions on D-branes and instantons, JHEP 10 (2008) 112, [arXiv:0807.1666].

[29] M. Billo' et. al., Non-perturbative effective interactions from fluxes, JHEP 12 (2008) 102, [arXiv: 0807.4098].

[30] A. M. Uranga, D-brane instantons and the effective field theory of flux compactifications, JHEP 01 (2009) 048, [arXiv:0808.2918].

[31] M. Billo et. al., Classical gauge instantons from open strings, JHEP 02 (2003) 045, [hep-th/0211250].

[32] R. Argurio, M. Bertolini, S. Franco, and S. Kachru, Gauge/gravity duality and meta-stable dynamical supersymmetry breaking, JHEP 01 (2007) 083, [hep-th/0610212].

[33] R. Argurio, M. Bertolini, S. Franco, and S. Kachru, Metastable vacua and D-branes at the conifold, JHEP 06 (2007) 017, [hep-th/0703236].

[34] R. Argurio, M. Bertolini, G. Ferretti, A. Lerda, and C. Petersson, Stringy Instantons at Orbifold Singularities, JHEP 06 (2007) 067, [arXiv:0704.0262].

[35] L. E. Ibanez, A. N. Schellekens, and A. M. Uranga, Instanton Induced Neutrino Majorana Masses in CFT Orientifolds with MSSM-like spectra, JHEP 06 (2007) 011, [arXiv: 0704.1079].

[36] R. Blumenhagen, M. Cvetič, R. Richter, and T. Weigand, Lifting D-Instanton Zero Modes by Recombination and Background Fluxes, JHEP 10 (2007) 098, [arXiv:0708.0403].

[37] C. Beasley and E. Witten, New instanton effects in supersymmetric QCD, JHEP 01 (2005) 056, [hep-th/0409149].

[38] C. Beasley and E. Witten, New instanton effects in string theory, JHEP 02 (2006) 060, [hep-th/0512039].

[39] M. Cvetič, I. Garcia-Etxebarria, and J. Halverson, Global F-theory Models: Instantons and Gauge Dynamics, arXiv:1003.5337.

[40] R. Blumenhagen, B. Jurke, T. Rahn, and H. Roschy, Cohomology of Line Bundles: A Computational Algorithm, arXiv:1003.5217.

[41] S. Jow, Cohomology of toric line bundles via simplicial Alexander duality, arXiv:1006.0780.

[42] T. Rahn and H. Roschy, Cohomology of Line Bundles: Proof of the Algorithm, arXiv:1006.2392.

[43] M. Cvetič, R. Richter, and T. Weigand, Computation of D-brane instanton induced superpotential couplings - Majorana masses from string theory, Phys. Rev. D76 (2007) 086002, [hep-th/0703028]. 
[44] S. Antusch, L. E. Ibanez, and T. Macri, Neutrino Masses and Mixings from String Theory Instantons, JHEP 09 (2007) 087, [arXiv:0706.2132].

[45] R. Blumenhagen, M. Cvetič, D. Lust, R. Richter, and T. Weigand, Non-perturbative Yukawa Couplings from String Instantons, Phys. Rev. Lett. 100 (2008) 061602, [arXiv:0707.1871].

[46] M. Cvetič, J. Halverson, and R. Richter, Realistic Yukawa structures from orientifold compactifications, JHEP 12 (2009) 063, [arXiv:0905.3379].

[47] M. Cvetič, J. Halverson, and R. Richter, Mass Hierarchies from MSSM Orientifold Compactifications, JHEP 07 (2010) 005, [arXiv: 0909.4292].

[48] M. Cvetič, J. Halverson, and R. Richter, Mass Hierarchies vs. Proton Decay in MSSM Orientifold Compactifications, arXiv:0910.2239.

[49] M. Cvetič, J. Halverson, P. Langacker, and R. Richter, The Weinberg Operator and a Lower String Scale in Orientifold Compactifications, arXiv:1001.3148.

[50] E. Sharpe, Lectures on D-branes and sheaves, hep-th/0307245.

[51] M. Cvetič and T. Weigand, Hierarchies from D-brane instantons in globally defined Calabi-Yau Orientifolds, Phys. Rev. Lett. 100 (2008) 251601, [arXiv:0711. 0209].

[52] J. J. Heckman, J. Marsano, N. Saulina, S. Schafer-Nameki, and C. Vafa, Instantons and SUSY breaking in F-theory, arXiv:0808.1286.

[53] J. Marsano, N. Saulina, and S. Schafer-Nameki, An Instanton Toolbox for F-Theory Model Building, JHEP 01 (2010) 128, [arXiv:0808.2450].

[54] I. Garcia-Etxebarria and A. M. Uranga, Non-perturbative superpotentials across lines of marginal stability, JHEP 01 (2008) 033, [arXiv:0711.1430].

[55] A. M. Turing, On Computable Numbers, with an Application to the Entscheidungsproblem, Proc. London Math. Soc. 42 (1936), no. 1 230-265.

[56] K. Weihrauch, Computable Analysis. Springer, Berlin, 2000.

[57] R. Donagi and M. Wijnholt, Model Building with F-Theory, arXiv:0802.2969.

[58] C. Beasley, J. J. Heckman, and C. Vafa, GUTs and Exceptional Branes in F-theory - I, JHEP 01 (2009) 058, [arXiv:0802.3391].

[59] C. Beasley, J. J. Heckman, and C. Vafa, GUTs and Exceptional Branes in F-theory - II: Experimental Predictions, JHEP 01 (2009) 059, [arXiv:0806.0102].

[60] R. Donagi and M. Wijnholt, Breaking GUT Groups in F-Theory, arXiv:0808.2223.

[61] T. Weigand, Lectures on F-theory compactifications and model building, arXiv:1009.3497.

[62] A. Collinucci, New F-theory lifts, JHEP 08 (2009) 076, [arXiv:0812.0175].

[63] A. Collinucci, New F-theory lifts II: Permutation orientifolds and enhanced singularities, JHEP 04 (2010) 076, [arXiv: 0906. 0003].

[64] R. Blumenhagen, T. W. Grimm, B. Jurke, and T. Weigand, F-theory uplifts and GUTs, JHEP 09 (2009) 053, [arXiv: 0906. 0013].

[65] M. Cvetič, I. Garcia-Etxebarria, and R. Richter, Branes and instantons at angles and the F-theory lift of O(1) instantons, AIP Conf. Proc. 1200 (2010) 246-260, [arXiv:0911.0012].

[66] R. Donagi and M. Wijnholt, MSW Instantons, arXiv:1005.5391. 
[67] E. Witten, Non-Perturbative Superpotentials In String Theory, Nucl. Phys. B474 (1996) 343-360, [hep-th/9604030].

[68] D. Forcella, I. Garcia-Etxebarria, and A. Uranga, E3-brane instantons and baryonic operators for D3-branes on toric singularities, JHEP 03 (2009) 041, [arXiv:0806.2291].

[69] J. Marsano, N. Saulina, and S. Schafer-Nameki, Monodromies, Fluxes, and Compact Three-Generation F-theory GUTs, JHEP 08 (2009) 046, [arXiv:0906.4672].

[70] R. Blumenhagen, T. W. Grimm, B. Jurke, and T. Weigand, Global F-theory GUTs, Nucl. Phys. B829 (2010) 325-369, [arXiv:0908.1784].

[71] T. W. Grimm, S. Krause, and T. Weigand, F-Theory GUT Vacua on Compact Calabi-Yau Fourfolds, JHEP 07 (2010) 037, [arXiv:0912.3524].

[72] T. W. Grimm and T. Weigand, On Abelian Gauge Symmetries and Proton Decay in Global F- theory GUTs, arXiv:1006.0226.

[73] J. Marsano, N. Saulina, and S. Schafer-Nameki, A Note on G-Fluxes for F-theory Model Building, arXiv:1006.0483.

[74] Y. Matiyasevich, Hilbert's 10th Problem. The MIT Press, 1993.

[75] S. Yau, A survey of calabi-yau manifolds, in Surveys in differential geometry. Vol. XIII. Geometry, analysis, and algebraic geometry: forty years of the Journal of Differential Geometry, vol. 13, pp. 277-318. Int. Press, Somerville, MA, 2009.

[76] M. R. Douglas and S. Kachru, Flux compactification, Rev. Mod. Phys. 79 (2007) 733-796, [hep-th/0610102].

[77] R. Blumenhagen, V. Braun, T. W. Grimm, and T. Weigand, GUTs in Type IIB Orientifold Compactifications, Nucl. Phys. B815 (2009) 1-94, [arXiv:0811.2936].

[78] K. Oguiso, On algebraic fiber space structures on a Calabi-Yau 3-fold. Appendix by Noburu Nakayama., Int. J. Math. 4 (1993), no. 3 439-465.

[79] S. Kachru and C. Vafa, Exact results for $N=2$ compactifications of heterotic strings, Nucl. Phys. B450 (1995) 69-89, [hep-th/9505105].

[80] P. Candelas, X. De La Ossa, A. Font, S. H. Katz, and D. R. Morrison, Mirror symmetry for two parameter models. I, Nucl. Phys. B416 (1994) 481-538, [hep-th/9308083].

[81] D. R. Morrison and C. Vafa, Compactifications of F-Theory on Calabi-Yau Threefolds - I, Nucl. Phys. B473 (1996) 74-92, [hep-th/9602114].

[82] F. Denef and M. R. Douglas, Computational complexity of the landscape. I, Annals Phys. 322 (2007) 1096-1142, [hep-th/0602072].

[83] S. G. Williamson, Lattice Multiverse Models, arXiv:1009.2058.

[84] S. Franco, A. Hanany, K. D. Kennaway, D. Vegh, and B. Wecht, Brane Dimers and Quiver Gauge Theories, JHEP 01 (2006) 096, [hep-th/0504110].

[85] K. D. Kennaway, Brane Tilings, Int. J. Mod. Phys. A22 (2007) 2977-3038, [arXiv: 0706.1660].

[86] M. Yamazaki, Brane Tilings and Their Applications, Fortsch. Phys. 56 (2008) 555-686, [arXiv:0803.4474]. 
[87] M. Bianchi and E. Kiritsis, Non-perturbative and Flux superpotentials for Type I strings on the $Z_{3}$ orbifold, Nucl. Phys. B782 (2007) 26-50, [hep-th/0702015].

[88] M. Bianchi, F. Fucito, and J. F. Morales, D-brane Instantons on the $T^{6} / Z_{3}$ orientifold, JHEP 07 (2007) 038, [arXiv:0704.0784].

[89] P. G. Camara, E. Dudas, T. Maillard, and G. Pradisi, String instantons, fluxes and moduli stabilization, Nucl. Phys. B795 (2008) 453-489, [arXiv:0710.3080].

[90] L. E. Ibanez and R. Richter, Stringy Instantons and Yukawa Couplings in MSSM-like Orientifold Models, JHEP 03 (2009) 090, [arXiv:0811.1583].

[91] C. Angelantonj, C. Condeescu, E. Dudas, and M. Lennek, Stringy Instanton Effects in Models with Rigid Magnetised D-branes, Nucl. Phys. B818 (2009) 52-94, [arXiv:0902.1694].

[92] M. Bianchi, F. Fucito, and J. F. Morales, Dynamical supersymmetry breaking from unoriented D-brane instantons, JHEP 08 (2009) 040, [arXiv:0904.2156].

[93] M. Billo et. al., Stringy instanton corrections to N=2 gauge couplings, JHEP 05 (2010) 107, [arXiv: 1002 .4322].

[94] P. G. Camara, C. Condeescu, E. Dudas, and M. Lennek, Non-perturbative Vacuum Destabilization and D-brane Dynamics, JHEP 06 (2010) 062, [arXiv: 1003.5805].

[95] S. Franco et. al., Dimers and Orientifolds, JHEP 09 (2007) 075, [arXiv: 0707. 0298].

[96] L. E. Ibanez and A. M. Uranga, Instanton Induced Open String Superpotentials and Branes at Singularities, JHEP 02 (2008) 103, [arXiv:0711.1316].

[97] B. Feng, Y.-H. He, K. D. Kennaway, and C. Vafa, Dimer models from mirror symmetry and quivering amoebae, Adv. Theor. Math. Phys. 12 (2008) 3, [hep-th/0511287].

[98] A. Hanany, C. P. Herzog, and D. Vegh, Brane tilings and exceptional collections, JHEP 07 (2006) 001, [hep-th/0602041].

[99] N. Smart, The Algorithmic Resolution of Diophantine Equations. Cambridge University Press, Cambridge, 1998.

[100] K. A. Intriligator and N. Seiberg, Duality, monopoles, dyons, confinement and oblique confinement in supersymmetric SO(N(c)) gauge theories, Nucl. Phys. B444 (1995) 125-160, [hep-th/9503179].

[101] N. Akerblom, R. Blumenhagen, D. Lust, E. Plauschinn, and M. Schmidt-Sommerfeld, Non-perturbative SQCD Superpotentials from String Instantons, JHEP 04 (2007) 076, [hep-th/0612132].

[102] D. Cox, J. Little, and H. Schenck, Toric varieties, Amer. Math. Soc. Providence, RI, to appear. http://www.cs.amherst.edu/ dac/toric.html.

[103] W. Stein et. al., Sage Mathematics Software (Version 4.5). The Sage Development Team, 2010. http://www.sagemath.org.

[104] V. Braun and A. Novoseltsev, "Toric Geometry in the Sage CAS." in preparation.

[105] W. Fulton and J. Harris, Representation Theory : A First Course (Graduate Texts in Mathematics / Readings in Mathematics). Springer, July, 1999.

[106] L. B. Anderson, J. Gray, Y.-H. He, and A. Lukas, Exploring Positive Monad Bundles And A New Heterotic Standard Model, JHEP 02 (2010) 054, [arXiv:0911.1569]. 